WILHELM KEMPF

\title{
Psychologische Forschung als Begegnung
}

\section{Einleitung}

Seit einem runden Jahrhundert versteht sich die Psychologie als Erfahrungswissenschaft. Ich habe Zweifel daran, wie weit die Psychologie auch nur eines der beiden Worte - „Erfahrung“ und „Wissenschaft“ - zu Recht für sich in Anspruch nimmt. Diese Zweifel scheinen mir zumindest so weit berechtigt, als in der Psychologie Tendenzen bemerkbar sind, ihr Selbstverständnis als Wissenschaft an einer bestimmten - und selbst nicht mehr als begründungsbedürftig angesehenen - Methodik festzumachen ${ }^{1}$ und/oder überhaupt nur noch zur Kenntnis zu nehmen, was in das Korsett dieser Methodik paßt. Unter dem Verlust eines den gesamten Wissenschaftsprozeß umfassenden Methodologieverständnisses (vgl. Blumer 1973) hat sich in der Psychologie zudem der Glaube daran eingenistet, „Wissenschaftlichkeit" sei ein Wert für sich. Die Frage, wozu es denn gut sein soll, wissenschaftliche Psychologie zu betreiben, ist mehr und mehr aus dem Blickfeld gerückt. Vor dem Hintergrund solcher Vorstellungen halte ich z. B. die Diskussion um quantitative oder qualitative Methoden der Psychologie für fruchtlos. Ich möchte daher ein Methodologieverständnis vorschlagen, das keine Methode der Psychologie von vorneherein als ,wissenschaftlich" auszeichnet, sondern sich auf den Ursprung des Wortes „Methode“ besinnt.

Das Wort „Methode“ kommt bekanntlich aus dem Griechischen und bedeutet so viel wie "der Weg zu etwas". Wissenschaft hat Ziele und sucht nach geeigneten Wegen, diese Ziele zu erreichen. Die Wege sind in unserem Fall die Methoden der Psychologie. Das Ziel ist die Bildung eines Wissens über etwas, das als wissenswert erachtet wird. Je nachdem, was wir in der Psychologie als wissenswert erachten, werden wir entsprechend verschiedener Methoden bedürfen. Hinter dem Streit darum, was zum Methodeninventar der Psychologie gehören soll und was nicht, steht in Wahrheit oft ein Interessenkonflikt darum, was für uns wissenswert ist, oder darum, welche Art von Erfahrungen mit Hilfe der Psychologie zugänglich gemacht werden soll. 
Erfahrung kann vielerlei bedeuten. In der experimentellen Psychologie haben wir es mit einem sehr engen Erfahrungsbegriff zu tun. Die Erfahrungen, die hier gemacht werden, sind auf die Bestätigung oder Verwerfung vorgefaßter Meinungen - sogenannter „Hypothesen“ beschränkt. Wirklich tiefgreifende Erfahrungen (die - im Ernstfall das Leben des Forschers selbst verändern könnten) sind durch die Distanz zwischen Forscher und Forschungsobjekt - der „Versuchsperson" - so gut wie ausgeschlossen. Nun gibt es in der Psychologie zweifellos eine Reihe von Fragestellungen - insbesondere im Bereich der Allgemeinen Psychologie - die sich aus dieser Distanz heraus bearbeiten lassen. Schlimm wird es erst, wenn die angesprochene Erfahrungsarmut der Psychologie zum methodischen Prinzip wird und genau darauf läuft der dogmatische Anspruch der messenden und experimentierenden Psychologie akademisch-psychologischer Provenienz letztlich hinaus. Denn „wissenschaftliche Psychologie“ bedeutet dann nicht mehr eine Verfeinerung und Erweiterung lebensweltlicher Erfahrungsmöglichkeiten, sondern deren Einschränkung. Sie dient dann nicht mehr dem Erkenntnisdrang des Menschen, sondern wird zum Werkzeug der Verdrängung.

Jeder Wissenschaft geht eine Entscheidung darüber voraus, was als wissenswert erachtet wird. Diese Entscheidung ist in der Psychologie keineswegs einheitlich ausgefallen. Es lassen sich zumindest drei idealtypische Konzeptionen von Psychologie rekonstruieren, die ich als das „behavioristische“, das „kognitive“ und das „humanistische“ Psychologieverständnis bezeichnet habe (Kempf 1983b).

Als behavioristisches Psychologieverständnis möchte ich dabei eine Konzeption von Psychologie kennzeichnen, die sich in Methodologie und Methodik am Vorbild der Naturwissenschaften orientiert und den Sinn wissenschaftlichen Tuns in dessen technischer Verwertbarkeit sieht. Ersichtlich ist dieses Verständnis von Psychologie von dem Streben danach geprägt, in derselben Weise Macht über menschliches Verhalten zu gewinnen, wie wir sie in vielen Bereichen gegenüber der unbelebten Natur schon gewonnen haben. Als „behavioristisches Psychologieverständnis" bezeichne ich es, weil es vor allem Behavioristen wie Watson, Skinner oder Buss sind, denen das Verdienst zukommt, eine naturwissenschaftliche Auffassung von Psychologie konsequent ausformuliert, in ein wissenschaftstheoretisches Programm übersetzt und damit auch kritikfähig gemacht zu haben. In methodischer Hinsicht ist das behavioristische Psychologieverständnis durch dreierlei gekennzeichnet: (1) die Beschränkung auf das beobachtbare Verhalten; (2) die Erklärung von Verhaltensweisen mittels Deduktion aus empirischen Gesetzmäßigkeiten und objektiv bestimmbaren Stimulusbedingungen; und (3) Experimentiertechniken und Mathematik als Methoden schlechthin. 
$\mathrm{Da} ß$ vieles von dem, was die akademische Psychologie seither unter ihrem naturwissenschaftlichen Selbstverständnis treibt, mit diesem wissenschaftstheoretischen Programm kaum etwas zu tun hat, ist die durchaus tragische Folge des geringen Augenmerks, welches die akademische Psychologie methodologischen Fragen schenkt. „Tragisch“ deshalb, weil sie die Grundlage dafür bildet, daß auch die vielgerühmte „kognitive Wende“ in der Psychologie nicht etwa einen Paradigmenwechsel nach sich gezogen hat, sondern man wurstelt so weiter wie bisher, nur daß man keine methodologischen Skrupel mehr hat, wenn in der Theoriesprache intentionale Prädikatoren vorkommen, Worte wie „Gedanken“, „Gefühle“ oder „Vorstellungen“.

Was ich hier als das kognitive Psychologieverständnis charakterisieren möchte, stimmt denn mit dem methodologischen Selbstverständnis der meisten Vertreter der kognitiven Psychologie keineswegs überein, wenngleich es in der kognitiven Psychologie eine ganze Reihe von Theorien - insbesondere strukturelle Lerntheorien und Computersimulationstheorien - gibt, die sich einschließlich der ihnen zugehörigen experimentellen Forschung unter diesem Wissenschaftsverständnis weit angemessener darstellen lassen als unter den gängigen Naturwissenschaftlichkeitsansprüchen ( $\mathrm{vgl}$. dazu insbesondere Landa 1969, Scandura 1973, Scandura \& Brainerd 1978). Hauptsächlich handelt es sich hier jedoch um jenes Wissenschaftsverständnis, das der (z.T. von der konstruktiven Wissenschaftstheorie der Erlanger Schule beeinflußten) handlungstheoretischen Kritik an der akademischen Psychologie zugrundeliegt (vgl. dazu insbesondere Aschenbach 1981, 1982; Brandtstädter 1981; Hilke 1982; Kempf 1978, 1981, 1982a, 1983b; Smedslund 1976, 1978a, 1978b, 1980; Werbik 1981). Wissenschaft wird unter dieser Konzeption von Psychologie als ein aufgabenorientiertes Handeln verstanden, wobei die technische Verwertbarkeit von Wissen dann nur noch einen Spezialfall darstellt. Dabei wird ein Wissenschaftsverständnis, das (immer noch) auf die zielgerichtete Veränderung des Menschen abhebt, mit einer anthropologischen Konzeption verbunden, die durch Stichworte wie „Mündigkeit" und „Eigenverantwortlichkeit" gekennzeichnet ist.

In methodischer Hinsicht ist dieses kognitive Psychologieverständnis gekennzeichnet durch (1) eine Akzentverschiebung vom (distanziert-objektiv) beobachtbaren Verhalten auf gleichwohl mittels objektivierbarer Deutungsregeln aus dem Verhalten rekonstruierbare Sinngehalte sowie (2) durch die Aufgabe des deduktiv-nomologischen Erklärungsschemas: empirische Korrelationen zwischen Stimulusbedingungen und Verhaltensweisen werden nicht mehr als Erklärung des Verhaltens hingenommen, sondern nur noch als Beschreibung erklärungsbedürftiger Zusammenhänge aufgefaßt. Als Erklärungsprinzip dient der praktische Syllogismus (vgl. von Wright 1974), der 
sich grob etwa so zusammenfassen läßt: „Wenn ich in bestimmter Weise denke und gemäß meinem Denken handle, dann werde ich am Ende ein bestimmtes Verhalten zeigen". Entsprechend verändert sich damit (3) die Funktion des Experimentes, das nun nicht mehr dazu dient, empirische Gesetzmäßigkeiten zu überprüfen, sondern die methodisch geregelte Rekonstruktion von Denkinhalten und Denkabläufen ermöglichen soll.

Als „humanistisch" möchte ich dagegen eine Konzeption von Psychologie charakterisieren, die sich von den vorgenannten insofern radikal unterscheidet, als es ihr nicht so sehr um ein zielgerichtetes Eingreifen geht, sondern die ihr Sinnkriterium in der Verständnisbildung sieht. Die technische Verwertbarkeit von Wissen ist als Relevanzkriterium psychologischer Forschung endgültig zugunsten dessen in den Hintergrund getreten, was Holzkamp (1972) als „emanzipatorische Relevanz" bezeichnet. Dahinter steht - ähnlich wie im symbolischen Interaktionismus - die bis auf Epiktet zurückverfolgbare Auffassung, daß es nicht die Dinge ${ }^{2}$ selbst sind, die die Menschen bewegen, sondern die Ansichten, die sie von ihnen haben, oder, wie dies Blumer (1973, p. 81) ausgedrückt hat, „daß Menschen ,Dingen' gegenüber auf der Grundlage der Bedeutungen handeln, die diese für sie besitzen ". ${ }^{3}$ Entsprechend ist das Erkenntnisinteresse der humanistischen Psychologie nicht so sehr dadurch gekennzeichnet, daß wir gleichsam objektive Aussagen über Menschen gewinnen wollen, sondern dadurch, daß wir die Subjektivität des Menschen nachzuvollziehen und in Sinnzusammenhänge einzuordnen versuchen. Dazu gehört auch: die gesellschaftliche Vermitteltheit individueller Subjektivität transparent zu machen. Denn, wie Blumer (1973) zu Recht feststellt, werden Bedeutungen den Dingen nicht einfach appliziert, sondern die Bedeutung der Dinge ist aus der sozialen Interaktion abgeleitet, die mensch mit seinen Mitmenschen eingeht, und wird in der Auseinandersetzung mit den ihm begegnenden Dingen zugleich gehandhabt und abgeändert. Insofern ist eine recht verstandene humanistische Psychologie stets auch Sozialpsychologie im eigentlichen Sinne des Wortes. Verständnisbildung als Erkenntnisinteresse, unter dem Psychologie betrieben wird, impliziert, daß viele der von den Naturwissenschaften übernommenen Wissenschaftlichkeitskriterien traditioneller empirischer Sozialforschung noch radikaler überdacht werden müssen, als dies schon vom kognitiven Psychologieverständnis nahegelegt wird. Der einschneidende Unterschied zwischen auf Erklärung menschlichen Verhaltens ausgerichteter, traditioneller empirischer Sozialforschung und auf Verständnisbildung ausgerichteter, interpretativer Sozialforschung beginnt bereits dort, wo wir danach fragen, welche Funktion Theorie denn überhaupt im Forschungsprozeß zukommt. 
Im traditionellen Paradigma dient Theorie als Deduktionsbasis für Hypothesen, d.h. sie nimmt empirische Wirklichkeit vorweg, dient dazu, daß aus ihr Annahmen über die empirische Wirklichkeit abgeleitet werden können. Im interpretativen Paradigma dient Theorie als Interpretationsfolie, d. h. sie liefert eine Perspektive, unter der empirische Wirklichkeit geordnet werden kann. Dies hat Auswirkungen darauf, wie mit Theorie wissenschaftlich umzugehen ist: Theorie als Deduktionsbasis ist daraufhin zu befragen, ob sie auch empirisch wahrt ist. An Theorie als Interpretationsfolie haben wir die Frage zu stellen, wie leistungsfähig sie im Hinblick auf die ihr zukommende Ordnungsaufgabe ist. Als Deduktionsbasis konzipierte Theorien können einander widersprechen. Dem Umgang mit ihnen ist eine puristische (entweder/oder) Haltung angemessen. Als Interpretationsfolie konzipierte Theorien eröffnen allenfalls Perspektiven, die nicht gleichzeitig eingenommen werden können. Dem Umgang mit ihnen ist eine dialektische Haltung angemessen.

Mit dem beim Übergang vom traditionellen zum interpretativen Paradigma vollzogenen Funktionswandel von Theorie geht zugleich ein Wandel der Funktion einher, welche die Empirie zu erfüllen hat: Als Deduktionsbasis konzipierte Theorien bedürfen zu ihrer Überprüfung der systematischen Herstellung und Variation gezielter Ausschnitte der empirischen Wirklichkeit, des Experiments, in dem nur zum Tragen kommen soll, was auch in der Theorie vorkommt. Die von Holzkamp (1972) konstatierte zunehmende Desintegration und Parzellierung psychologischer Forschung ist daher nicht nur Folge der Präzisierung und Verfeinerung einer bestimmten Art von Methodik - nämlich der einseitigen Betonung von Design-und Meßtechniken, welche den Bestätigungsgrad empirischer Hypothesen optimieren sollen - sondern die Bevorzugung des Bestätigungsgrades empirischer Hypothesen als Kriterium für den Wert wissenschaftlicher Forschungsbemühungen im traditionellen Paradigma ist ihrerseits die konsequente Folge des ihm zugrundeliegenden Theorieverständnisses. Als Interpretationsfolie konzipierte Theorien bedürfen zu ihrer Anwendung dagegen der Explikation und Rekonstruktion möglichst umfassender Ausschnitte der "natürlichen“ empirischen Wirklichkeit. Im interpretativen Paradigma ist es daher die Strukturähnlichkeit zwischen Forschungssituation und Alltagssituation (respektive Anwendungssituation), der als Kriterium für den Wert wissenschaftlicher Forschungsbemühungen eine ähnlich bevorzugte Stellung zukommt wie dem Bestätigungsgrad empirischer Hypothesen im traditionellen Paradigma. 5 


\section{Drei Zugangsweisen zur Frustrations-Aggressions-Theorie}

Im weiteren Verlauf dieser Arbeit wird es vor allem um das humanistische Psychologieverständnis gehen. Die Existenzberechtigung anderer Konzeptionen von Psychologie soll dadurch keineswegs in Frage gestellt werden. Ich bin allerdings der Auffassung, daß die verschiedenen Psychologieverständnisse hierarchisch geordnet sind, daß vom humanistischen über das kognitive hin zum naturwissenschaftlichbehavioristischen Psychologieverständnis eine zunehmende methodologische Reduktion stattfindet, die auf ihre Angemessenheit für die jeweilige Fragestellung zu beurteilen ist. Dabei sollten wir stets im Auge behalten, daß uns diese Reduktion zwar desto „härtere“ Methoden anbietet, je weiter sie getrieben wird, daß sie unseren Blickwinkel aber zugleich auf immer enger werdende Ausschnitte der menschlichen Existenz einengt. Wie ich am Beispiel der FrustrationsAggressions-Theorie deutlich machen möchte, hat dies zur Folge, daß es innerhalb der Psychologie jedenfalls einige Fragestellungen gibt, die unter bestimmten Psychologieverständnissen nicht mehr angemessen thematisiert werden können, bzw. die methodologischen Forderungen dieser Psychologieverständnisse bei der Bearbeitung jener Fragestellungen nicht eingelöst werden können.

Inhaltlich der Psychoanalyse nahestehend, zugleich aber im Bemühen um eine streng behavioristische Methodologie formuliert, umfaßt die 1939 von der Yale-Gruppe (Dollard, Doob, Miller, Mowrer \& Sears) vorgestellte Frustrations-Aggressions-Theorie neben den Definitionen

- D1.l: Aggression ist eine Verhaltenssequenz, die auf die Verletzung (injury) eines Organismus oder Organismusersatzes abzielt; und

- D2.1: Frustration ist die Störung einer zielgerichteten Aktivität; die beiden Grundannahmen

- Al.l: Aggression ist stets eine Folge von Frustration; und

- A2.1: Frustration führt stets zu einer Form von Aggression; von denen letztere allerdings bald durch Miller (1941) und Sears (1941) abgeschwächt wurde:

- A2.1*: Frustration erzeugt Anreize zu verschiedenen Arten von Verhaltensweisen; einer dieser Anreize ist stets ein Anreiz zu einer Form von Aggression.

Ferner umfaßt die Theorie eine Reihe von Zusatzannahmen, von denen vor allem die sogenannte Katharsis-Hypothese einige Berühmtheit erlangt hat:

- A3.l: Durch die Ausführung einer Aggression wird der von der Frustration erzeugte Anreiz zur Aggression reduziert; der Ar...-- 
zur Fortsetzung der gestörten Verhaltenssequenz bleibt dabei weiter bestehen.

Damit wurde eine Erklärung aggressiven Verhaltens im Sinne des deduktiv-nomologischen Erklärungsmodells der Naturwissenschaften angestrebt. Dieses Erklärungsmodell kann im sogenannten HempelOppenheim-Schema (vgl. Hempel 1965, Stegmüller 1969) so dargestellt werden, daß der zu erklärende Sachverhalt (das „Explanandum") E aus einer Reihe von Gesetzesaussagen G1, G2, ..., Gm und Randbedingungen R1, R2,.., Rn (die zusammen das „Explanans“ bilden) deduziert wird:

$$
\begin{aligned}
& \mathrm{G} 1, \mathrm{G} 2, \ldots, \mathrm{Gm} \\
& \mathrm{R} 1, \mathrm{R} 2, \ldots, \mathrm{Rn} \\
& \mathrm{E}
\end{aligned}
$$

Bei den Gesetzesaussagen handelt es sich dabei um universelle Subjunktionen der Form $\wedge_{\mathrm{x}} \mathrm{A}(\mathrm{x}) \rightarrow \mathrm{B}(\mathrm{x})$, wobei $\mathrm{A}(\mathrm{x})$ als das "Antecedens" („unabhängige Variable“) und $B(x)$ als das „Succedens“ (,abhängige Variable“) der Gesetzesaussage bezeichnet wird. Ist ein solcher Allsatz wahr, so kann man dann, wenn $A(N)$ für ein individuelles $N$ zutrifft, vermittels $\wedge x A(x) \rightarrow B(x) . A(N)>B(N)$ auf das Bestehen von $B(N)$ schließen und derart das Explanandum $B(N)$ aus der Gesetzesaussage $\wedge{ }_{x} A(x) \rightarrow B(x)$ und der Randbedingung $A(N)$ erklären. ${ }^{6}$ Damit eine Deduktion nach dem Hempel-Oppenheim-Schema tatsächlich als eine Erklärung gelten kann, müssen noch einige Voraussetzungen erfüllt sein (vgl. dazu z. B. Stegmüller 1969, von Kutschera 1972, Schwemmer 1976). Insbesondere: (a) Sowohl im Antecedens als auch im Succedens der Gesetzesaussagen dürfen ausschließlich empirische Aussagen vorkommen, d.h. nur solche Aussagen, deren Wahrheit oder Falschheit von der Beobachtung abhängt und nicht schon allein aufgrund logischer, terminologischer oder mathematischer Regeln beweisbar ist; sowie (b) die den zu erklärenden Sachverhalt darstellenden Aussagen dürfen nicht schon aufgrund logischer, terminologischer oder mathematischer Regeln aus den Aussagen ableitbar sein, die die jeweiligen Antecedensbedingungen darstellen.

Zweifel daran, ob die von der Yale-Gruppe vorgelegte FrustrationsAggressions-Theorie diese methodologischen Forderungen auch tatsächlich erfüllen kann, wurden zunächst im Hinblick auf die zugrundeliegende Aggressions-Definition laut. So hat insbesondere Buss (1961) kritisiert, daß Aussagen über Verhaltensintentionen nicht durch Beobachtung (allein) entscheidbar seien. Sein Versuch, diese Verletzung des behavioristischen Methodenideals zu heilen, führte zwar zur Erfindung der „Aggressionsmaschine“, einem experimentallan 
Paradigma, das den Mangel an Strukturübereinstimmung zwischen Alltagssituation und Experiment geradezu potenziert, konnte jedoch den intentionalen Gehalt des Aggressionsbegriffes dennoch nicht völlig eliminieren: selbst wenn Buss definiert „... aggression is defined as a response that delivers noxious stimuli to another organism" (1961, p. 1), bleibt für ihn doch die Frage offen, wie zufällig gelieferte, schädliche Stimuli von Aggressionen abgegrenzt werden können (vgl. Hilke \& Kempf 1976). Hinzu kommt, daß auch das Wort „schädlich“ kein beobachtungssprachlicher Prädikator ist, sondern der Beurteilungssprache angehört. Die Besinnung auf den intentionalen Gehalt des Aggressionsbegriffes (Werbik 1971) - aber auch die auf Buss zurückgehende Unterscheidung zwischen „angry aggression“ und ,instrumentally aggressive responses" führten schließlich zur Frage, ob und wieweit denn die in der Frustrations-Aggressions-Theorie formulierten Gesetzesaussagen tatsächlich empirischen Gehalt haben oder sich einfach aus der Art und Weise ergeben, wie wir über Handeln im allgemeinen und über Frustration, Aggression und Katharsis im speziellen sprechen (vgl. Smedslund 1976).

Am detailliertesten ausgearbeitet wurde in diesem Zusammenhang ein Rekonstruktionsversuch der Frustrations-Aggressions-Theorie für instrumentelle Aggressionen (Kempf 1978), der freilich von einem sehr allgemeinen Aggressionsbegriff ausgeht und nicht mehr auf die Verletzung eines Organismus abhebt, sondern (wie bei Buss) nur noch auf dessen Schädigung, und zwar in einem sehr vordergründigen Sinne: Schädigung als Verstoß gegen den Willen eines anderen:

- D-1.2: Aggression ist eine Handlung, die (nach Meinung des Handelnden) gegen den Willen eines anderen verstößt.

- D-2.2: Frustration ist ein Ereignis, als dessen Folge eine Handlung (in Hinblick auf die damit verfolgten Handlungsorientierungen) erfolglos bleibt.

- D-3.2: Wenn Handlungsorientierungen einander wechselseitig be-oder verhindern, so besteht ein Konflikt.

Daraus ergibt sich unmittelbar:

- A-1.2: Aggression setzt voraus, daß (nach Meinung des Handelnden) ein Konflikt besteht.

- A-2.2a: Tritt eine Frustration als (tatsächliche oder vermeintliche Wirkung des Handelns eines anderen ein, und deute ich diese Wirkung als vom anderen gewollt, so besteht (nach meiner Meinung) ein Konflikt.

- A-2.2b: Halte ich in diesem (tatsächlichen oder vermeintlichen) Konflikt an meinen ursprünglichen Handlungsorientierungen fest und versuche. diese gegen den (tatsächlichen oder vermeintlichen) Willen des anderen durchzusetzen, so sind alle darauf gerichteten Handlunoon meinerseits per definitionem Aggressionen. 
- A-3.2: Behalte ich meine ursprünglichen Handlungsorientierungen bei und setze sie erfolgreich durch (d.h. sind die von mir verfolgten Handlungsziele am Ende erreicht), so entfällt der Grund zur Aggression (d.h. jene Handlungsziele, deren Verfolgung mit der Frustration und ihrer Deutung als vom anderen intendiert zu aggressiven Zielen geworden sind, bestehen nicht mehr).

Infolge der vorgenommenen Differenzierung zwischen Frustration und Konflikt werden die ursprünglichen Annahmen der FrustrationsAggressions-Theorie durch diese Rekonstruktion zwar modifiziert. Diese Modifikation macht es jedoch möglich, die strukturellen und die empirischen Anteile der Theorie voneinander zu trennen: Die von der Frustrations-Aggressions-Theorie behauptete Regelmäßigkeit. mit der Frustrationen aggressiv beantwortet werden, ist an die materialen Voraussetzungen (1) der Deutung der Frustration als intendierter Wirkung des Handelns eines anderen und (2) des Festhaltens an den ursprünglichen Handlungsorientierungen geknüpft; jedes nichtaggressive Handeln in einer Frustrationssituation setzt voraus, daß ich entweder die Frustration nicht als intendierte Wirkung des Handelns eines anderen deute oder daß ich meine ursprünglichen Handlungsorientierungen aufgebe (bzw. zumindest vorläufig zurückstelle).

Was in der Frustrations-Aggressions-Theorie als einfache empirische Regelmäßigkeit zwischen Stimulusbedingungen und Verhaltensweisen konzipiert worden war, wirft damit eine Reihe von neuen Fragen auf, zu deren Behandlung Aggression nicht mehr als isoliertes Phänomen - gleichsam losgelöst von dem historischen und gesellschaftlichen Kontext, in dem Aggressionen stattfinden - betrachtet werden kann. Z. B. stellt sich die Frage, wie sich jene subjektive Realität konstituiert, die Aggression zur einzig angemessenen Antwort auf eine Frustration macht und dabei ursprünglich instrumentelle Aggression oft in feindselige Aggression umschlagen läßt, bei der es nicht mehr nur darum geht, ursprüngliche Handlungsorientierungen durchzusetzen, sondern (auch) darum, den anderen zu verletzen (im Sinne der Definition der Yale-Gruppe).

Die Antwort auf solche Fragen sprengt nicht nur den Rahmen des beobachtungssprachlich Beschreibbaren, sie kann auch nicht mehr auf der Sprachebene von finalen Handlungsorientierungen gegeben werden, die auf mehr oder minder objektiv beschreibbare Handlungsziele gerichtet sind, mit deren Erreichung sie gleichsam „aufgehoben werden". Denn bei diesen Fragen geht es nun nicht mehr um einzelne Verhaltensweisen, Handlungen oder Handlungsorientierungen in unserem Leben, sondern es geht um den Gesamtzusammenhang unseres Handelns und Lebens und darum, welche Bedeutung eine Frustration und die uns verfügbaren Handlungsmöglichkeiten, vermittels 
welcher wir auf die Frustration antworten könnten, in diesem Gesamtzusammenhang gewinnen. Es geht nicht mehr um gleichsam objektive Beziehungen zwischen Frustration und Aggression, sondern um individuelle, aber gleichwohl gesellschaftlich vermittelte Subjektivität. Es geht darum, wie sich mensch in seinem Leben orientiert, wie sich die objektiven (sozialen) Umwelt-Gegebenheiten - das soziale Milieu - zur sozialen Situation konstituiert. „Die Umwelt kann auf das Subjekt nur in genau dem Maße einwirken, in dem dieses sie versteht, d.h. indem dieses sie in Situationen verwandelt" (Sartre 1956, p. 720). Erst dadurch, wie wir uns in einem Milieu orientieren, wird das Milieu zur Situation. Die soziale Situation konstituiert sich als Interaktionspraxis zwischen von der Situation betroffenen Individuen und Milieugegebenheiten (vgl. Leithäuser \& Volmerg 1977).

Kambartel $(1978,1981)$ hat in Anlehnung an Wittgenstein den Terminus „Lebensorientierungen“ geprägt und damit eine Orientierungsebene angesprochen, die sich im Hinblick auf die zu ihrer Darstellung erforderlichen sprachlichen Mittel grundlegend von der Ebene der Handlungsorientierungen unterscheidet. Im Unterschied zu den Handlungsorientierungen sind Lebensorientierungen afinal, d.h. sie weisen einen Weg, der sich nicht vom Ende her bestimmen läßt. Und, wir begreifen Lebensformen letztlich weniger theoretisch als vielmehr im ihnen gemäßen Erleben und Handeln, also „empraktisch". Worte genügen erst, wenn eine Lebensform bereits empraktisch zugänglich ist, sie zu vergegenwärtigen. Wenn die Worte, mit denen wir über Lebensformen reden, dieser empraktischen Basis entbehren, dann helfen uns noch so viele theoretische, insbesondere definitorische Bemühungen nicht weiter. Über Lebensformen läßt sich daher auch nicht in derselben Weise argumentieren wie über Handlungsorientierungen. Wer eine Lebensform ein Stück weit empraktisch begriffen hat, dem mag es gelingen, mit anderen (die sich in derselben Lage befinden) einen Konsens darüber herzustellen. Eine Kritik „von außen“ ist dagegen wenig hilfreich. Lebensorientierungen sind daher auch nicht in derselben Weise verfügbar wie Handlungsorientierungen. Die Aufgabe oder Modifikation von Lebensorientierungen gelingt - wenn überhaupt - so nur um den Preis einer (zumindest vorübergehenden) Identitätskrise. Denn in ihnen sind all jene Selbstverständlichkeiten unseres Erlebens und Handelns niedergelegt, die es uns überhaupt erst erlauben, unser Leben als ganzes und uns selbst als identische Person zu verstehen. 


\section{Lebensorientierungen, Gesellschaftscharakter und Alltagsbewußtsein}

Mit den Lebensorientierungen wird wie bei Fromms „Charakterorientierungen“ eine Orientierungsebene angesprochen, die den Menschen grundsätzlich vom Tier unterscheidet, denn nur für ihn stellt sich aufgrund seiner hoch ausgebildeten Sprach- und Denkfähigkeit das existentielle Problem der Sinngebung des Lebens. Gleichwohl sind Lebensorientierungen - im Unterschied zu konkreten Handlungszielen - weitgehend unbewußt und können daher auch nicht im Paradigma traditioneller empirischer Sozialforschung - etwa durch direkte Befragung - erfaßt werden. Sie sind der subjektiven Realität, welche sie konstituieren, implizit und erfordern zu ihrer Bewußtmachung und Explikation daher einen eigenen Reflexions- bzw. Interpretationsprozeß.

Darauf weist auch Fromm (1962) hin, indem er die Unzulänglichkeiten des Charakterbegriffs der traditionellen empirischen Sozialforschung kritisiert, die den Charakter als das für ein bestimmtes Individuum charakteristische Verhaltensmuster definiert. Fromm beruft sich dabei auf Freuds dynamischen Charakterbegriff, der

„... den Charakter als ein System von Strebungen auffaßte, die dem Verhalten zugrundeliegen, jedoch nicht mit ihm identisch sind... Verhaltensmerkmale beziehen sich auf Tätigkeiten, die von einer dritten Person beobachtet werden können... Wenn wir jedoch die Motivationen und insbesondere die unbewußten Motive solcher Verhaltensmerkmale untersuchen, so finden wir, daß das Verhaltensmerkmal zahlreichen, völlig unterschiedlichen Charakterzügen entspricht... Freud hat etwas erkannt, was die großen Romanschriftsteller und Dramatiker schon immer wußten: daß - wie Balzac sich ausdrückt - das Charakterstudium , sich mit den Kräften befaßt. die den Menschen motivieren", daß die Art und Weise, wie jemand handelt, fühlt und denkt, weitgehend durch die Besonderheit seines Charakters bestimmt ist, und da 3 sie nicht nur das Resultat rationaler Reaktionen auf bestimmte Situationen ist. Freud erkannte die dynamische Qualität der Charakterzüge und stellte fest, daß die Charakterstruktur eines Menschen eine spezielle Form darstellt, in der die Energie im Lebensprozeß kanalisiert wird" (Fromm 1962, p. 85-87).

Die Charakterzüge eines Menschen sind mehr als nur (mehr oder minder) stabile Merkmale seines Handelns und Verhaltens. Es ist die Art und Weise, wie sich jemand in seinem Leben orientiert, die seinen Charakter ausmacht. Es ist der Charakter eines Menschen, der bestimmt, wie er den Gegenständen der belebten und unbelebten Natur gegenübertritt, welche Bedeutung die Ereignisse seiner Umwelt für ihn haben, wie er gefühlsmäßig auf sie reagiert, welche Handlungswünsche sie in ihm wachrufen, wie er mit seinen Gefühlen und Bedürfnissen umgeht und wonach er in seinem Leben strebt. Die Frage nach dem Charakter eines Menschen, das ist die Frage nach den Orientierungen seines Lebens, welche ihm eine über seine jeweiligen 
sozialen Rollen und seine physischen und psychischen Eigenschaften hinausreichende Identität verleihen. Es ist letztlich die Frage, wer er denn eigentlich sei (vgl. Kempf 1982b), und diese Frage läßt sich im Grunde nur narrativ beantworten. Die Worte, mit denen wir die Lebensorientierungen eines Menschen beschreiben können, gewinnen ihre Bedeutung erst aus dem Kontext seines Lebens und werden erst verstehbar, wenn wir zugleich die konkreten Beispielsituationen angeben, welche durch sie in einen Sinnzusammenhang gebracht werden, und das heißt: indem wir die Subjektivität des anderen und deren gesellschaftliche Vermittlung zu rekonstruieren versuchen.

$\mathrm{Da} \beta$ Adorno et al. (1950) diese methodologischen Konsequenzen von Freuds dynamischem Charakterbegriff übersehen und den Versuch unternommen haben, die autoritätsgebundene Persönlichkeit mittels des Instrumentariums der traditionellen empirischen Sozialforschung - d.h. mittels Persönlichkeitsfragebögen - zu erfassen, führte am Ende in jene methodischen Aporien, an denen die Untersuchungen zur autoritätsgebundenen Persönlichkeit notwendigerweise scheitern mußten und deren Ursprung Leithäuser \& Volmerg (1977, p. 30) darin sehen, daß ihre Bezugsgröße das Individuum ist, „seine Charakterstruktur, die sich nach Auffassung Horkheimers und Adornos nicht sozialpsychologisch, sondern in letzter Instanz nur individualpsychologisch" aufschließen läßt. Indem der Charakter eines Menschen von Adorno derart doch wieder als „Summe individueller Persönlichkeitszüge im Verhalten und Handeln“ (Argelander 1972) aufgefaßt wird, erscheinen die Vergesellschaftungsprozesse, durch die die Individuen vermittelt sind, nur als Randbedingung der empirischen Untersuchung zur autoritätsgebundenen Persönlichkeit. Durch methodische Reduktion wird das Verhältnis von Individuum und Gesellschaft in einen abstrakten Gegensatz verwandelt. Und was erst künstlich getrennt wurde, muß dann wieder ebenso künstlich zusammengefügt werden. So läßt sich z. B. allein gestützt auf die empirischen Befunde der Studien, die die situativen Faktoren, die Milieufaktoren und die gesellschaftlichen Bedingungen durch ihre Erhebungsund Auswertungsmethoden notwendig vernachlässigen, die Vorurteilsbereitschaft als Strukturmerkmal des ,autoritären Charakters“ nicht begründen. Es bedarf zusätzlicher allgemeiner theoretischer Überlegungen, die die methodologische Reduktion auf die als individualpsychologische Kategorie aufgefaßten Charaktertypen rückgängig machen sollen. Zu diesem Zweck wird z. B. der gesellschaftliche Faktor der „ideologischen Gesamtstruktur" herangezogen, womit nichts anderes gemeint ist „als unser allgemeines kulturelles Klima und besonders die ideologische Wirkung, welche die Massenbeeinflussungsmittel auf die Bildung der öffentlichen Meinung ausüben“. Mit der Einführung von nicht an den Charakter gebundenen Größen 
wird aber, wie Leithäuser \& Volmerg (1977) aufzeigen, der autoritäre Charakter als individualpsychologische Bezugsgröße selbst unglaubwürdig: „Denn wie sollte erklärbar sein, daß die positiven Reaktionen auf die Skalensätze von einem Angehörigen der Mittelschicht auf dessen Charaktervariablen verweisen und die gleichen Reaktionen von einem Arbeiter auf dessen ökonomische Lage? Nach einer solchen Auflösung der Bezugsgröße gibt es keine gemeinsame Meßgrundlage mehr" (p. 35).

Anders als bei Adorno ist eine künstliche Trennung zwischen einem individualpsychologisch zu analysierenden Charakter und gesellschaftlichen Randbedingungen dagegen bei Fromm nicht von vornherein angelegt.? „Nach Marx besteht zwischen der ökonomischen Basis der Gesellschaft und den politischen und rechtlichen Institutionen sowie ihrer Philosophie, Kunst, Religion usw. eine wechselseitige Abhängigkeit. Nach der Marxistischen Theorie werden letztere - der ,ideologische Überbau' - von der ökonomischen Basis bestimmt. Aber Marx und Engels haben nicht gezeigt - was übrigens Engels ausdrücklich zugab -, wie die ökonomische Basis in den ideologischen Überbau übersetzt wird“ (Fromm 1962, p. 85). Diese Lücke will Fromm mittels des Charakterbegriffs schließen. D. h. der Charakterbegriff dient bei Fromm als eine jener Bezugsgrößen, mittels welcher die Vermittlung zwischen ökonomischer Basis und ideologischem Überbau analysiert wird. Dabei gewinnt die Charakterstruktur freilich erst dann über den Einzelmenschen hinaus an Bedeutung, „wenn sich nachweisen läßt, daß auch ganze Völker und Gesellscharten oder Klassen innerhalb einer Gesellschaft eine Charakterstruktur besitzen, die für sie charakteristisch ist, auch wenn die einzelnen Individuen sich auf vielfältige Weise voneinander unterscheiden, und es auch eine Anzahl von Menschen darunter geben wird, deren Charakterstrukturen überhaupt nicht in die umfassende Struktur der Gesamtgruppe hineinpassen“ (p. 89). Diese für eine Gesellschaft typische Orientierungsweise nennt Fromm den "Gesellschaftscharakter" und weist darauf hin, daß der Gesellschaftscharakter nicht statisch verstanden werden dürfe, „so als ob er die Gesamtsumme der bei der Mehrheit der Menschen in einer bestimmten Kultur anzutreffenden Charakterzüge darstellte. Er wird nur richtig verstanden, wenn er hinsichtlich seiner Funktion verstanden wird“ (p. 89). Zur Bestimmung der Funktion des Gesellschaftscharakters geht Fromm (1949, p. 210) davon aus, daß Aufbau und Handlungsweise einer Gesellschaft durch eine Anzahl objektiver Gegebenheiten notwendig werden.

"Solche Bedingungen sind die Produktionsweise und die Güterverteilung, welche ihrerseits von den Rohmaterialien und Herstellungstechniken, vom Klima usw. abhängen, sowie von politischen und geographischen Faktoren und kulturellen Traditionen und Einflüssen, denen die Gesellschaft ausgesetzt isl. 
Es gibt keine ,Gesellschaft ${ }^{\star}$ als solche, sondern nur bestimmte Gesellschaftsstrukturen ... Obgleich diese Gesellschaftsstrukturen im Lauf der Geschichte sich ändern. sind sie während eines bestimmten geschichtlichen Zeitabschnitts doch relativ beständig, und eine Gesellschaft kann nur bestehen, insofern sie sich innerhalb des Rahmens dieser bestimmten Struktur bewegt ... Die Aufgabe des Gesellschafts-Charakters besteht darin, die Energien der Mitglieder der Gesellschaft so zu formen, daß ihr Verhalten nicht mehr einer bewußten Entscheidung bedarf, ob sie sich dem Sozialgefüge einordnen sollen oder nicht; daß die Menschen vielmehr so handeln wollen, wie sie handeln müssen, und daß sie gleichzeitig darin eine Genugtuung finden... Der GesellschaftsCharakter formt die menschliche Energie so, daß sie das reibungslose Funktionieren einer gegebenen Gesellschaft garantiert".

Vergegenwärtigen wir uns, da $B$ der Gesellschaftscharakter aber nicht nur „die Energien der Mitglieder der Gesellschaft" in einer bestimmten Weise formt, sondern daß er dies tut, indem er eine bestimmte, für die Gesellschaft typische Art und Weise darstellt, wie sich Menschen in ihrem Leben orientieren, wie sich die objektiven (sozialen) Gegebenheiten zu gemeinsam geteilter subjektiver Realität konstituieren, so wird deutlich, daß der Gesellschaftscharakter nicht nur eine derart gesellschaftsstabilisierende Funktion besitzt. Er ist - indem er überhaupt erst gemeinsame soziale Realität schafft - konstitutiv für Gesellschaft schlechthin. Daß der Gesellschaftscharakter nur hinsichtlich seiner Funktion richtig verstanden werden kann, bedeutet, daß auch die empirische Analyse des Gesellschaftscharakters nicht auf jener bloß deskriptiven Ebene stehenbleiben kann, auf der die für eine Gesellschaft typischen Lebensorientierungen dargestellt werden, sondern daß gleichzeitig auf einer funktionalen Ebene aufzuweisen ist, wie diese Orientierungen der Aufrechterhaltung der Gesellschaftsstruktur und der innerhalb einer Gesellschaft bestehenden Herrschaftsverhältnisse dienen, d.h. insbesondere, wie das Milieu der Gesellschaft unter diesen Orientierungen in jene soziale Situation transformiert wird, in der die gegebene Gesellschaftsstruktur unter ihren objektiven Bedingungen als notwendig und diese Bedingungen ihrerseits als unverrückbar erscheinen. Oder anders ausgedrückt: es ist aufzuweisen, wie die zum Gesellschaftscharakter gehörigen Orientierungen die Struktur der Gesellschaft in der Identität ihrer Mitglieder verankert. ${ }^{8}$ Und es ist auf einer strukturellen Ebene empirisch aufzuweisen, mittels welcher Mechanismen die Funktionsweise dieser Orientierungen gesellschaftlich hergestellt und aufrechterhalten wird.

Für die Analyse des Gesellschaftscharakters auf dieser dritten Ebene ist der von Leithäuser (1978) geprägte Begriff des Alltagsbewußtseins von maßgeblicher Bedeutung, mit dem der gegenwärtigen Form der Vergesellschaftung des Bewußtseins Rechnung getragen werden soll. In seiner Theorie des Alltagsbewußtseins geht Leithäu- 
ser davon aus, daß die unmittelbaren Lebenszusammenhänge des Menschen durch die Vergesellschaftung von ihrer traditionalen Herkunft getrennt und dem Verwertungsinteresse des Kapitals fungibel gemacht werden. Das Verwertungsinteresse paßt sich an die wirklichen Bedürfnisse an, modelt sie aber gleichzeitig um, um sie in seine abstrakte Systematik einzupassen. Die Vergesellschaftung produziert einen permanenten Regressionsdruck in allen Lebensbereichen, durch welchen Übertragungsvorgänge erzeugt und verstärkt werden, mittels welcher die Individuen ihre Wahrnehmungen und Interpretationen von einer sozialen Situation zu anderen verknüpfen. Die Gesellschaft hat sich Institutionen geschaffen, die diese Übertragungsvorgänge unterstützen und erleichtern, gleichsam Übertragungsangebote bereitstellen. Unter dem Einfluß der Bewußtseinsindustrie kommt es zu einer Aufspaltung des Bewußtseins in ein alltagspraktisches, erfahrungsgebundenes Bewußtsein und ein industriell vororganisiertes Bewußtsein, bei dem sich Meinungen nicht in der bewußten Auseinandersetzung mit erkennbaren Sachverhalten bilden, sondern die öffentlich dargebotenen Symbole korrespondieren mit unbewußten, dem Einzelnen in ihrer Mechanik verborgenen Prozessen.

Innerhalb des Alltagsbewußtseins sind derart zwei Bewußtseinsmodi unterscheidbar, von denen der eine sozialisationsbedingt ist und auf Einsichten in die eigene Lebensgeschichte, auf einer (wie auch immer beschränkten) Reflexionsfähigkeit beruht, während der andere von den Medien vorgefertigt, gleichsam industriell produziert, ohne große individuelle Modifikationen und Erfahrungskumulation internalisiert wird. Die beiden Bewußtseinsmodi lagern nicht abstrakt nebeneinander (mit jeweils Dominanzen des einen über den anderen), sondern bestimmen sich wechselseitig, desgleichen auch die Sozialisationsagenturen, so daß eine Aufgliederung der Sozialisation in (aufeinanderfolgende) primäre und sekundäre Sozialisation schwierig wird. Deshalb kann auch nicht mehr davon ausgegangen werden, daß nur die Familie der Ort ist, an dem die uns interessierenden Orientierungen der Individuen hergestellt werden. Die Entwicklung der Bewußtseinsindustrie verschärft diese Verwischung der Grenzen zwischen den Sozialisationsphasen noch, indem die Massenmedien einerseits den Einfluß der traditionellen Sozialisationsagenturen zurückdrängen, aber zugleich jene Tendenzen verstärken, die regressiv an die Familienstruktur binden.

Die Funktionsweise des Alltagsbewußtseins ist durch Übertragungsprozesse geprägt, d. h. durch den bloß wiederholenden, auf Assimilation gerichteten Gebrauch von das Alltagsbewußtsein strukturierenden und die soziale Situation organisierenden Regeln angesichts fremder und neuer sozialer Situationen. Dadurch werden kumulative 
Prozesse, bei denen sich das Bewußtsein Stufe um Stufe mit Erfahrungen und Wissen anreichert, abgewehrt und auf nicht-kumulative Prozesse der einfachen Reproduktion reduziert. Die Übertragungsregeln (Abwehrregeln, Reduktionsregeln und Thematisierungsregeln) dienen der Aufrechterhaltung des durch Thema und Horizont gegliederten Bewußtseinsfeldes, des "Thema-Horizont-Schemas“. Unter dem Horizont des Alltagsbewußtseins versteht Leithäuser den Gesichtskreis, der all das umfaßt und umschließt, was von einem Punkt aus sichtbar ist, d.h. thematisch werden, einer bewußten Verarbeitung zugänglich gemacht werden kann. Das Alltagsbewußtsein erschließt sich keine neuen Horizonte. Aber auch nicht alles innerhalb des Horizonts des Alltagsbewußtseins kann zum relevanten Thema werden: Der Routinebereich des Alltagsbewußtseins ist nicht innerhalb des Horizonts des Alltagsbewußtseins thematisierbar. Aufgrund seiner internalisierten „Plausibilitätsstruktur“ (Berger \& Luckmann 1969) kann das Routinewissen nicht zur Sprache gebracht werden. Die Sinndeutungen, die im Rahmen des Thema-Horizont-Schemas die Orientierung der Individuen herstellen und aufrechterhalten, sind zwar potentiell kommunizierbar und thematisierbar, ihre Thematisierungschance wird jedoch durch internalisierte „Intersubjektivität“ minimiert, d.h. durch die internalisierte Unterstellung gegenseitigen Verstehens.

Intersubjektivität ist die lnstanz der Vergesellschaftung des Bewußtseins. Sie ist Resultat gesellschaftlicher Anpassungsprozesse, die sie zugleich garantieren muß, und wird durch Abwehr verteidigt, die den Horizont des Alltagsbewußtseins abdichtet; insbesondere durch Harmonisierung, d.h. durch die Schwächung der Thematisierungschance eines Konfliktes; durch Nivellierung, d. h. durch Löschung der Thematisierungschance; oder durch Exterritorialisierung, d.h. durch Verlagerung des Konfliktes außerhalb des Thema-Horizont-Schemas. Intersubjektivität konstituiert sich im Medium der Sprache und wird aufrechterhalten durch Gelegenheitsausdrücke, d. h. Wortausdrücke, bei denen der Sinn, den eine besondere Gelegenheit ihnen aufdrückt, ihren üblichen (sachlichen) Sinn dominiert; durch die „eigentümliche Vagheit", einen Überschuß an Bedeutungen im Alltagsdiskurs, der nicht aufzuheben ist und in der Interaktion immer mitgedacht werden muß; sowie durch die Unterstellung von Sinnübereinstimmung, aufgrund welcher in alltäglicher Kommunikation ein.Sprecher erwarten kann, daß der von ihm intendierte Sinn von den Angesprochenen in der gleichen Weise aufgefaßt wird, wie er gemeint war. Garfinkel (1973, p. 208) schreibt dazu: „Abweichungen von der allgemein üblichen Praxis, Gelegenheitsausdrücke zu benutzen, die unausweichliche Vagheit hinzunehmen sowie Sinneinverständnis zu beanspruchen (und zu unterstellen), rufen unmittelbare Versuche 
hervor, diese allgemein übliche Praxis als einen erwünschten $\mathrm{Zu}$ stand der Dinge wieder herzustellen“. Nach Leithäuser \& Volmerg (1977, p. 58) beschreiben solche Versuche „noch einmal die Intention des Alltagsbewußtseins, seinen Horizont mit der der sozialen Situation möglichst deckungsgleich zu halten“. Das Alltagsbewußtsein möchte reibungslosen Verkehr und Verlauf der sozialen Situationen sicherstellen, den status quo bewahren, beim Vertrauten und Bekannten bleiben, jede qualitative Umformung des Thema-HorizontSchemas vermeiden, indem es das Unbekannte auf das scheinbar Bekannte reduziert. Zu diesem Zweck funktionalisiert die Abwehr auch die Thematisierung selbst und stellt sie in ihren Dienst, indem sie nur geschwächte oder verdrehte Thematisierung zuläßt, die Thematisierung auf andere Konfliktzonen innerhalb des ThemaHorizont-Schemas verschiebt, oder sie außerhalb des Horizonts verlagert.

Für die empirische Analyse des Übertragungsvorganges ergeben sich derart drei Fragestellungen: 1. Wie wird ein Thema thematisiert? Die Antwort erhalten wir durch Explikation der Thematisierungsregeln. 2. Wie wird ein Thema modifiziert? Hier ist die Antwort die Explikation der Reduktionsregeln. 3. Welche Funktion hat eine Thematisierung? Hier folgt die Explikation der Abwehrregeln. Besondere Bedeutung gewinnt das Konzept des Alltagsbewußtseins bei der strukturellen Analyse des Gesellschaftscharakters unter den Bedingungen des Kulturkonfliktes, d.h. dann, wenn sich innerhalb einer Gesellschaft Ansätze zu einem potentiell kulturrevolutionären Orientierungswandel entwickeln.

Bereits Fromm (1949) hatte darauf hingewiesen, daß der Gesellschaftscharakter zwar grundsätzlich gesellschaftsstabilisierende Funktion besitzt. Ändern sich jedoch die äußeren Bedingungen so, daß sie nicht mehr zu dem herkömmlichen Gesellschaftscharakter passen, und die ihm entsprechende Gesellschaftsordnung grundlegende Bedürfnisse der Menschen - in einer wie auch immer transformierten Form - nicht mehr erfüllen kann, so wird er zu einem Element der Zersetzung. In welcher Weise dies geschieht, kann anhand der Übertragungsvorgänge des Alltagsbewußtseins näher analysiert werden. Wenn eine Gesellschaftsordnung grundlegende Bedürfnisse der Menschen vernachlässigt, so werden die Mitglieder dieser Gesellschaft erwartbarerweise versuchen, die Gesellschaftsordnung zu ändern. Dagegen versucht sich der Gesellschaftscharakter so lange als irgend möglich zu schützen, indem eine Thematisierung der Bedürfnisdefizite nicht oder nur verzerrt zugelassen wird, bzw. durch eine Verzerrung der Thematisierung von auf die defizitären Bedürfnisse gerichteten Orientierungen, z. B. indem sich die neuen Orientierungen ein Praxisfeld suchen, in dem sie kompensatorisch verfolgt werden kön- 
nen, während der Gesamtzusammenhang des Handelns und Erlebens im traditionellen Gesellschaftscharakter verhaftet bleibt.

Während der erste dieser Mechanismen der Abwehr eines drohenden Kulturkonfliktes dient, versucht der Gesellschaftscharakter mittels des anderen der beschriebenen Mechanismen, potentiell kulturrevolutionäre Orientierungsänderungen zu transformieren und für sich zu vereinnahmen. Während sich ein Kulturkonflikt durchaus noch im Medium des Alltagsbewußtseins artikulieren kann, setzen kulturrevolutionäre Orientierungsänderungen voraus, daß der Horizont des Alltagsbewußtseins gesprengt wird. Wo sich kulturrevolutionäre Orientierungsänderungen - jedenfalls bei einer Teilgruppe innerhalb einer Gesellschaft - durchzusetzen beginnen, verhindern die Übertragungsmechanismen des Alltagsbewußtseins eine angemessene Thematisierung des Kulturkonflikts. Stattdessen werden Übertragungsangebote bereitgestellt, die die Mitglieder der Gesellschaft noch stärker an den traditionellen Gesellschaftscharakter binden sollen. Mit der verzerrten Thematisierung des Kulturkonfliktes wird auf die potentiell kulturrevolutionären Orientierungen erneut ein Transformationsdruck ausgeübt. Indem sie sich nur in einem verdrehten Kontext artikulieren können, wird ihre Thematisierung verzerrt, und sie laufen Gefahr, doch noch vom traditionellen Gesellschaftscharakter vereinnahmt $z u$ werden und/oder in eine bloße - und bloß punktuelle - Gegenposition zu diesem abzugleiten.

\section{Traditionelles und interpretatives Paradigma}

Unser empirisches Interesse gilt den Lebensorientierungen von Menschen in ihrer gesellschaftlichen Vermitteltheit. Wegen der Implizitheit von Lebensorientierungen ist ihre empirische Analyse notwendigerweise interpretativ. Und zwar in zweierlei Hinsicht: Erstens können Lebensorientierungen nur mittels eines Interpretationsprozesses aus der subjektiven Realität erschlossen werden, welche sie konstituieren, und zweitens erfordert schon die Erfassung subjektiver Realität, d.h. die Erfassung der Bedeutungen, welche die Dinge für einen Menschen haben, einen Interpretationsprozeß.

Taylor (1971) hat darauf hingewiesen, daß unser Verständnis der Worte, mittels welcher wir Bedeutungen erschließen können, sich unausweichlich in einem hermeneutischen Zirkel bewegt und notwendigerweise kontextabhängig ist.

\footnotetext{
„Our actions are ordinarily characterized by the purpose sought and explained by desires, feelings, emotions. But the language by which we describe our goals, feelings, desires is also a definition of the meaning things have for us. The vocabulary defining meaning - words like 'terrifying'. 'attractive', - is linked with that describing feeling - 'fear', 'desire' - and that describing goais - 'safety", "possession"... An emotion term like 'shame', for instance, essentially refers us
} 
to a certain kind of situation, the 'shameful', or 'humiliating', and a certain mode of response, that of hiding oneself, of covering up, or else 'wiping out' the blot. That is, it is essential to this feeling being identified as shame that it be related to this situation and give rise to this type of disposition. But this situation in its turn can only be identified in relation to the feeling which it provokes; and the disposition is to a goal which can similarly not be understood without reference to the feelings experienced: the 'hiding' in question is one which will cover up with my shame; it is not the same as hiding from an armed pursuer; we can only understand, what is meant by 'hiding' here if we understand what kind of feeling and situation is being talked about. We have to be within the circle.

An emotion term like 'shame' can only be explained by reference to other concepts which in turn cannot be understood without reference to shame. To understand these concepts we have to be in a certain experience, we have to understand a certain language, not just of words, but also a certain language of mutual action and communication, by which we blame, exhort, admire, esteem each other. In the end we are in on this because we grow up in the ambit of certain common meanings. But we can often experience what it is like to be on the outside when we encounter the feeling, action and experiential meaning language of other civilization" (p. 34-35).

Während die Kulturanthropologie durch den drastischen Wechsel des kulturellen Hintergrundes seit jeher für die Andersartigkeit ihrer Forschungssubjekte sensibilisiert war (vgl. Hoffmann-Riem 1980), fiel es der Psychologie mangels entsprechender Erfahrungen vergleichsweise leichter, in jener alltagsweltlichen Einstellung internalisierter Intersubjektivität zu verharren, in der die Dinge ,in meiner Umwelt und der meiner Mitmenschen für uns die gleichen sind und grundsätzlich die gleiche Bedeutung haben" (Schütz \& Luckmann 1979, p. 27). Solange die Regelhaftigkeit einer Handlung derart als kontextunabhängig feststellbar gilt (vgl. Wilson 1973), verhilft der Rückgriff auf ein (vermeintlich) gemeinsames Wertsystem als nicht weiter thematisierter Interpretationsrahmen zur bedeutungsmäßigen „Feststellung“empirischer Daten. Zweifel an dieser Feststellbarkeit markieren den Ausgangspunkt der interpretativen Sozialforschung und ziehen weitreichende methodologische Konsequenzen nach sich:

.Verhaltensweisen lassen sich in dieser Konzeption von gesellschaftlicher Wirklichkeit zwar raum-zeitlich exakt erfassen... Ihre Bedeutungskomponente entzieht sich jedoch dem objektivierenden Zugriff: Die Bedeutung einer sprachlichen Äußerung zum Beispiel ist indexikal"(vgl. Garfinkel \& Sacks 1973), „d. h. sie ist an den Kontext der Äußerung, etwa an die Intention von Sprecher und Hörer und deren Beziehung zueinander, wie an einen Index gebunden und losgelöst von diesem Kontext nicht faßbar. Da eine Äußerung nach Garfinkel nicht von ihrer Indexikalität, geheilt" werden kann, ohne daß ihr Sinn verfehlt wird, kann Sozialforschung die soziale Wirklichkeit auf ihrer ,empirischen" Ebene nur geleitet durch das Prinzip der Offenheit aufnehmen: Die aktuelle Konstitution von Bedeutung ist an die Kommunikationssituation gebunden, auch an die Kommunikationssituation des Forschungshandelns, und es gilt abzuwarten, wie sich Bedeutungszuschreibungen kontextabhängig herausbilden" (Hoffmann-Riem 1980, p. 344). 
Die theoretische Strukturierung des Forschungsgegenstandes muß zurückgestellt werden, bis sich die Strukturierung des Forschungsgegenstandes durch die Forschungssubjekte herausgebildet hat. Dieser Verzicht auf eine „Hypothesenbildung ex ante“ (Hoffmann-Riem) betrifft freilich nur die Vorwegnahme empirischer Wirklichkeit qua empirische Allaussagen, wie sie im deduktiv-nomologischen Erklärungsmodell getroffen wird. Auch interpretative Sozialforschung kann nicht theoriefrei getrieben werden, soll sie nachprüfbaren Kriterien für die Sicherung sozialwissenschaftlicher Erkenntnis gerecht werden.

Blumer (1973, p. 121) hat darauf hingewiesen, daß ,kein Theoretisieren, wie geistreich es auch sein mag, und keine Beachtung des wissenschaftlichen Programms, wie peinlich genau sie auch sein mag" ein „Ersatz für die Entwicklung einer Vertrautheit mit dem“ sind, „was tatsächlich in dem zu untersuchenden Lebensbereich vor sich geht“. Gleichzeitig muß aber auch in Rechnung gestellt werden, daß mensch die empirische Welt immer nur durch ein Schema oder eine Vorstellung von ihr wahrnehmen kann. „Die gesamte wissenschaftliche Untersuchung ist nach dem ihr zugrundeliegenden Bild der empirischen Welt ausgerichtet und von ihm gestaltet. Dieses Bild legt die Auswahl und Formulierung von Problemen fest, die Bestimmung der Dinge, die als Daten betrachtet werden, die für die Sammlung der Daten benutzten Mittel, die zwischen den Daten gesuchten Arten der Beziehungen und die Formen, in denen Vorschläge gemacht werden“ (Blumer 1973, p. 105). Dies ist das Spannungsfeld, in dem sich interpretative Sozialforschung notwendigerweise bewegt, und das die von Blumer getroffene Unterscheidung zwischen Exploration und Inspektion bedingt.

"Die explorative Erforschung des menschlichen Zusammenlebens ist das Mittel, um gleichzeitig zwei sich ergänzende und miteinander verbundene Ziele zu erreichen. Einerseits ist sie der Weg, über den ein Forscher eine enge und umfassende Bekanntschaft mit einem Bereich des sozialen Lebens herstellen kann, der ihm nicht vertraut und daher unbekannt war. Andererseits ist sie das Mittel, um seine Untersuchung zu entwerfen und zu verbessern, so daß seine Probleme, seine Untersuchungsausrichtung, seine Daten, seine analytischen Beziehungen und seine Interpretationen aus dem zu untersuchenden empirischen Leben hervorgehen und in ihm begründet bleiben" (Blumer 1973, p. 122)

Explorative Forschung ist daher notwendigerweise eine flexible Vorgehensweise, die noch nicht so strengen methodischen Kriterien gerecht werden kann, wie sie an die Inspektion anzulegen sind. Da eine ausgearbeitete Interpretationsfolie noch nicht verfügbar ist bzw. erst in Verlaufe der Exploration entwickelt wird, stellt die Exploration besondere Anforderungen an die Bereitschaft des Forschers, die seinen Sinndeutungen impliziten Prämissen zu reflektieren, wodir.h 
zugleich auch Selbsterfahrungsprozesse auf seiten des Forschers impliziert werden. Schon deshalb kann interpretative Sozialforschung nicht mit derselben Distanz zum Forschungsgegenstand betrieben werden, die das traditionelle Paradigma aufrechtzuerhalten sucht. Die Lebenswelt eines anderen wird für mich nur nachvollziehbar, wenn ich zugleich Klarheit über meine eigene Lebenswelt gewinne. Denn: Anerkennung und Verständnis fremder Subjektivität ist mir nur in dem Maße möglich, in dem ich eben meine eigene Subjektivität als solche erkenne" (Kempf 1983b, p. 17). Während die Exploration weitgehend deskriptiv bleibt, geht die Inspektion darüber hinaus:

\begin{abstract}
„Die direkte Prüfung der empirischen Welt ist nicht auf die Erstellung umfassender und vertrauter Darstellungen des Geschehens begrenzt. Sie sollte auch die Analyse beinhalten. Der Forscher, der eine direkte Prüfung vornimmt, sollte darauf hinzielen, sein Problem in eine theoretische Form zu gießen, die allgemeinen Beziehungen aufzudecken, die situationsspezifischen Bezüge seiner Konzepte zu schärfen und theoretische Entwürfe zu formulieren. Solch eine Analyse ist das eigentliche Ziel der empirischen Wissenschaft, wie sie sich von der Bereitstellung rein deskriptiver Darstellungen unterscheidet " (Blumer 1973, p. 125)
\end{abstract}

Die dazu erforderlichen theoretischen Sätze haben jedoch nicht den Status von empirischen Allgemeinaussagen, sondern sie haben den Status von methodischen Prinzipien, unter denen das Typische empirisch aufweisbar wird. In diesem Sinne sind etwa die im vorangegangenen Abschnitt skizzierten Übertragungsprozesse, welche die Funktionsweise des Alltagsbewußtseins prägen, nicht so zu verstehen, daß damit unterstellt würde, daß unser Bewußtsein ganz allgemein nach solchen Mechanismen funktioniert (was eine ziemlich unsinnige Unterstellung wäre), sondern diese Übertragungsprozesse definieren erst, was unter einer , alltagsweltlichen Einstellung“ gemeint ist, in der eben unser Bewußtsein durch solche Mechanismen dominiert ist und damit überhaupt erst zu dem wird, was Leithäuser als „Alltagsbewußtsein“ bezeichnet. Daraus folgt, daß auch die ers1 noch zu formulierenden Kriterien für die Sicherung sozialwissenschaftlicher Erkenntnis im Rahmen des interpretativen Paradigmas einen anderen Status haben müssen als die Kriterien zur Sicherung der „objektiven Erkenntnis“ im Rahmen des traditionellen Paradigmas. Sie sind nicht als Anleitungen zum Testen von Hypothesen, sondern als Anleitungen zur Interpretation zu verstehen.

Wie die Arbeitsgruppe Bielefelder Soziologen (1973. p. 43) hervorhebt, wurde innerhalb des interpretativen Paradigmas dem Problem der Kontrolle der Interpretitionen des Forschers lange Zeit nicht genügend Aufmerksamkeit geschenkt. „So kann es sicherlich nicht genügen anzunehmen, der Forscher werde nach einer gewissen Zeit des Zusammenlebens mit der untersuchten Gruppe .gültige Interpre- 
tationen' erbringen, die mit denen der Handelnden übereinstimmen es müssen auch Regeln entwickelt werden, nach denen eine Überprüfung dieser Interpretationen durch andere Forscher ermöglicht wird, und es müssen des weiteren Regeln für den Wechsel zwischen Innenperspektive und äußerer Analyse angegeben werden“. Leithäuser \& Volmerg (1977) haben in Analogie zu dem von Mertens (1975) aufgestellten Schema zur „Sicherung objektiver Erkenntnis“ ein Schema zur „Sicherung gültiger Interpretationen“ entwickelt, an dem sowohl die Gemeinschaft als auch die Unterschiedlichkeit der Gütekriterien deutlich wird, welche an sozialwissenschaftliche Erkenntnis im traditionellen Paradigma und im interpretativen Paradigma anzulegen sind (vgl. Abb. 1).

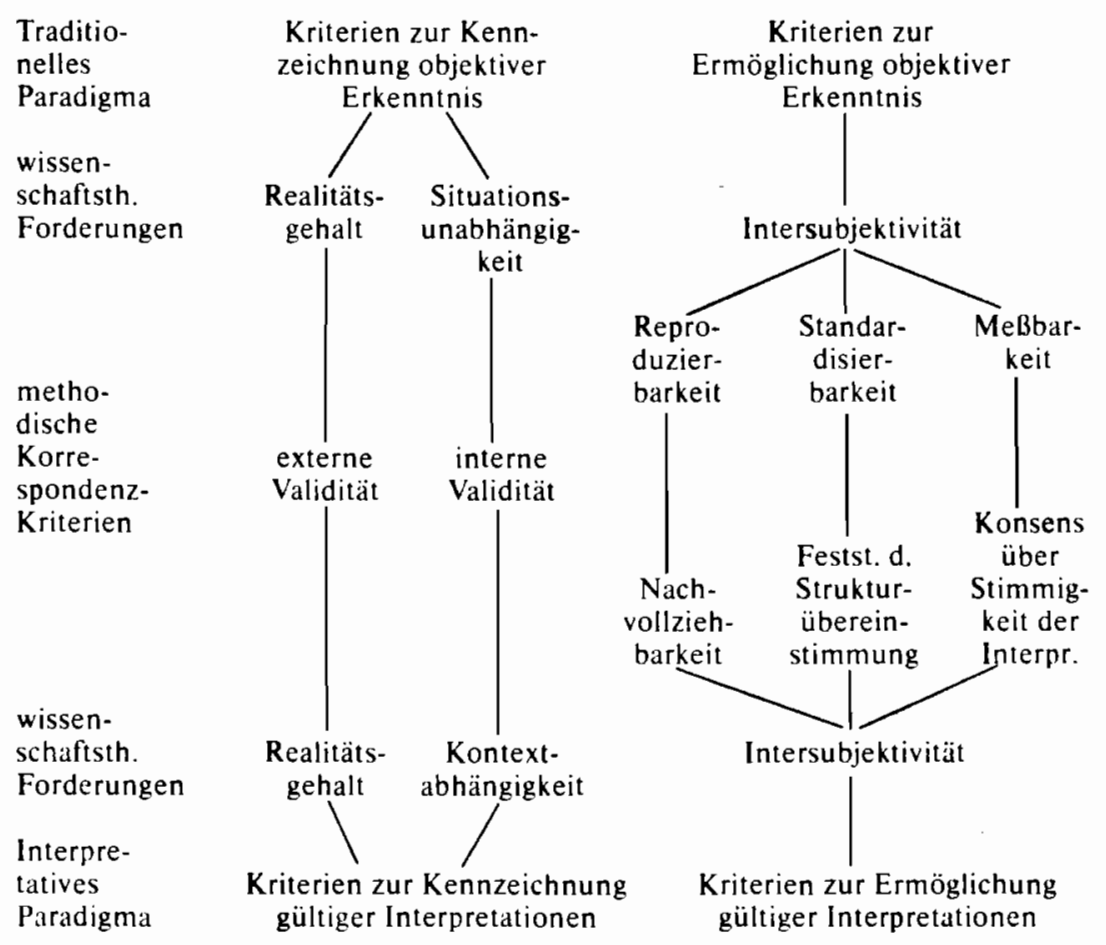

Ahh. I: Krilerien für die Sicherung sozialwissenschaftlicher Erkenntnis im Iraditionellen und im interpretativen Paradigma (nach Leithäuser \& Volmerg 1977)

Während die wissenschaftstheoretischen Forderungen nach Realitätshaltigkeit und Intersubjektivität von beiJen Paradigmen geteilt werden, ist die - im traditionellen Paradigma von den Naturwissenschaften übernommene - Forderung nach Situationsunabhängigkeit wegen der Indexikalität seines Forschungsgegenstandes (Garfinkel) 
im interpretativen Paradigma durch die Forderung nach Kontextabhängigkeit ersetzt. Dies bedeutet, daß interne Validität interpretativer Sozialforschung einerseits die gelungene praktische Teilhabe des Forschers an den mehr oder weniger impliziten Regulationen der sozialen Situationen erfordert, die untersucht werden sollen. Analog zu Lorenzers (1974) Unterscheidungen der psychoanalytischen Erkenntnis bezeichnen Leithäuser \& Volmerg (1977) die praktische Teilhabe des Forschers an und in der betroffenen sozialen Situation als ,hermeneutisches Feld I“. „Je korrekter und intensiver dem Forscher die praktische Teilhabe gelingt, umso besser erfüllt er das ,zugeordnete methodische Kriterium der internen Validität der engen Verknüpfung seiner wissenschaftlichen Erfahrung mit den Gegebenheiten des Untersuchungsfeldes“ (p. 131). Zugleich kann die interne Validität interpretativer Sozialforschung aber nur über die Konstruktion eines „hermeneutischen Feldes II" sichergestellt werden, denn man kann den Interpretationen und Berichten der Forscher aus dem hermeneutischen Feld I nicht einfach Glauben schenken:

„Sie müssen nachvollziehbar, ihre Gültigkeit muß überprüfbar sein ... - nicht als eines Forschungsinstrumentes, sondern im Sinne einer Kontrolle der gelungenen praktischen Teilhabe in sozialen Situationen. Im ,hermeneutischen Feld II' gelten andere Regeln als im ,hermeneutischen Feld I'. Während in diesem nur praktische Teilhabe, das Sich-Ausrichten auf und Sich-Anpassen an implizite (meist implizite) Regelsysteme. Verregelung möglich ist, die es nachträglich zu explizieren gilt, dominiert im ,hermeneutischen Feld Il von vorne herein die Nötigung zur Explikation der Regeln“ (Leithäuser \& Volmerg 1977, p. (31-132).

Im hermeneutischen Feld II ist über die Regelexplikation die Intersubjektivität der sozialwissenschaftlichen Erkenntnis herzustellen, wofür die - auf „Bedingungskontrolle“ (für psychologische Experimente) ausgerichteten - methodischen Korrespondenzkriterien für die Intersubjektivitätsgarantie im traditionellen Paradigma - Reproduzierbarkeit, Standardisierbarkeit und Meßbarkeit - jedoch nicht einfach übernommen werden können. An ihre Stelle treten Nachvollziehbarkeit, Feststeilung der Strukturübereinstimmung und Konsensbildung über die Stimmigkeit der Interpretation in der gleichen Rangfolge. Ihr Status muß freilich in jedem neuen Forschungszusammenhang in einem jeweils neuen hermeneutischen Feld II neu konstituiert werden. Nachvollziehbarkeit, Feststellung der Strukturübereinstimmung und Konsensbildung über die Stimmigkeit der Interpretationen sind Kriterien, über die sich die beteiligten Forscher in jedem Falle neu zu einigen haben, und keine Verfahrensanleitung. ${ }^{9}$

Der Unterscheidung zwischen den hermeneutischen Feldern I und Il entsprechend. wird vom Forscher die Anwendung zweier Perspektiven verlangt, einer "Innenperspektive“, wie es die Arbeitsgruppe 
Bielefelder Soziologen ausdrückt, und einer „äußeren Perspektive“. Die „Innenperspektive“ zielt auf die zu untersuchende Interaktionspraxis der sozialen Situation und betrifft die praktische Teilhabe des Forschers. ${ }^{10}$ Die „äußere Perspektive“ zielt auf die Regeln der Regelexplikation, „also auf die Interaktionspraxis der Forschergruppe im ,hermeneutischen Feld II", an der der Feldforscher teilnimmt" (Leithäuser \& Volmerg 1977, p. 134).

\section{Narratives Interview und partnerzentriertes Gespräch}

Dem zugrundeliegenden Theorieverständnis entsprechend, steht im traditionellen Paradigma der Bestätigungsgrad empirischer Hypothesen im Vordergrund des methodischen Kontrollinteresses. Dies hat zur Folge, daß die methodischen Korrespondenzkriterien der Intersubjektivität hauptsächlich durch die Forderung nach interner Validität geprägt sind. während die externe Validität - da sich Bestätigungsgrad empirischer Hypothesen und Strukturübereinstimmung zwischen Forschungssituation und Alltags- bzw. Anwendungssituation gegenseitig ausspielen 11 - allenfalls nachträglich und bloß korrelativ festgestellt werden kann.

In interpretativen Paradigma ist der Gegensatz zwischen externer und interner Validität dagegen nicht so kraß, wenngleich die Forderung nach methodischer Kontrolle empirischer Forschung auch hier immer noch in einem Spannungsverhältnis zur Realitätshaltigkeit der Erhebungssituation steht. Dies hat zur Folge, daß die Methoden der Datenerhebung im interpretativen Paradigma nicht schematisch gewählt werden können, sondern im Hinblick auf die jeweilige Fragestellung und im Hinblick auf die anvisierte Analyseebene jeweils neu zu bestimmen sind. In diesem Sinne können z. B. Leithäuser \& Volmerg (1977) davon ausgehen, daß für die empirische Analyse der Übertragungsprozesse des Alltagsbewußtseins die Realitätshaltigkeit der Erhebungssituation erfüllt ist, sofern sich eine gewisse Dichte von Vermittlungen gesellschaftlicher Strukturen und die häufige Wiederholung der Situation aufweisen lassen, d. h. wenn die Erhebungssituation in wesentlichen Strukturmerkmalen mit der typischen Alltagssituation übereinstimmt.

Bei der empirischen Analyse des Gesellschaftscharakters haben wir auf drei unterschiedlichen Analyseebenen zu operieren, die entsprechend auch eine unterschiedliche Qualität des empirischen Materials verlangen. Für die strukturelle Analyse der Mechanismen des Alltagsbewußtseins, mittels welcher die Funktionsweise der für den Gesellschaftscharakter typischen Orientierungen gesellschaftlich hergestellt und aufrechterhalten wird, haben Leithäuser et al. (1977) bereits umfassende methodologische Überlegungen angestellt, und es braucht 
darauf hier nicht näher eingegangen zu werden. Für die Analyse der Orientierungen des Gesellschaftscharakters in ihrer Funktion zur Aufrechterhaltung bestehender Herrschaftsverhältnisse erscheint es mir von besonderer Wichtigkeit, auch die von den gesellschaftlichen Institutionen unterbreiteten Übertragungsangebote mit einzubeziehen, wie ich dies in Kempf $(1984,1985)$ über die Analyse der Äußerungen politischer Meinungsführer zur Frage „Was heißt für dich Frieden?" und der Presseberichterstattung über die Friedensbewegung ansatzweise versucht habe. Eine methodologische Systematisierung dieser Analyseebene steht freilich noch aus und kann auch im Rahmen der vorliegenden Arbeit (noch) nicht geleistet werden.

Hier sollen lediglich einige methodologische Überlegungen zur deskriptiven Analyse von Lebensorientierungen angestellt werden. Dazu möchte ich zunächst in Erinnerung rufen, daß mit den Lebensorientierungen eine Orientierungsebene angesprochen ist, die nicht direkt abgefragt, sondern nur über einen eigenen Interpretationsprozeß zugänglich gemacht werden kann. Lebensorientierungen sind interpretative Kategorien, die individuelles Handeln und Erleben in einen Sinnzusammenhang stellen, indem sie jene Bedeutungen konstituieren, welche sich letztlich nur aus kontextabhängigen Handlungs- und Situationsbeschreibungen erschließ3en lassen. Damit ist auf eine von Schwemmer (in diesem Band) getroffene Unterscheidung verwiesen, wonach uns (mindestens) zwei grundlegend verschiedene Arten der Handlungsbeschreibung offenstehen.12 Die eine besteht darin. die Handlung als Verwirklichung eines Tätigkeitsmusters ${ }^{13}$ darzustellen. Die andere beschreibt eine Handlung als Teil einer Geschichte.

Eine Handlungsbeschreibung, die die Bedeutung des Handelns nicht übergehen will, ist niemals bloß die Beschreibung einer isolierten Tätigkeit, sondern immer eine Gesamtbeschreibung der Handlung in ihrem Kontext, und wie Schwemmer (in diesem Band) deutlich macht, sind auch die ,schlichten“ schematischen Handlungsbeschreibungen keine kontextfreien Beschreibungen, sondern nur solche Beschreibungen, deren Kontext nicht reflektiert ist, und die die Handlungen meist in einen anderen Kontext rücken als der es war, in dem sie von den handelnden Personen ausgeführt worden sind. It Als Ausgangspunkt für die empirische Erfassung von Lebensorientierungen reichen schematische Handlungsbeschreibungen daher keinesfalls aus. Der Frage nach jenen Orientierungen, weiche den Gesamtzusammenhang unseres Handelns und Erlebens herstellen, kann erst auf der Grundlage kontextbezogener Handlungsbeschreibungen nachgegangen werden. d.h. auf der Grundlage des Verständnisses jener Geschichte. die sich für das handeInde Subjekt tatsächlich abgespielt hat. ${ }^{15}$ 
Daraus folgt, daß wir einen empirischen Zugang zu den Lebensorientierungen von Menschen am ehesten über die Ausnutzung der alltagspraktischen Kompetenz des Erzählens gewinnen können, wie sie Schütze (1976, 1982, 1983) mit der Methode des ,narrativen Interviews" für die sozialwissenschaftliche Forschung zu nutzen versucht. Im Gegensatz zum geschlossenen Interview handelt es sich dabei um ein Verfahren. das auf eine vorhergehende Strukturierung der Interviewinhalte weitgehend verzichtet. Ein Leitfaden ist zwar vorhanden, „seine Bedeutung schrumpft jedoch durch die Eigenleistung der Forschungssubjekte" (Hoffmann-Riem 1980, p. 360) Durch den Verzicht auf eine vorherige Strukturierung, die den Interviewten auf vorherbestimmte Interviewinhalte festlegt, und das stattdessen intendierte „Hervorlocken von Geschichten" soll einerseits ein Maximum an Kontext geschaffen werden, um subjektive Realität interpretativ erfassen zu können, und andererseits soll erreicht werden, daß die Erzählung durch die Bedeutungshierarchie des Gesprächspartners strukturiert wird. Entsprechend empfiehlt Schütze eine Zweiteilung des Interviews in eine Haupterzählung und eine Phase des narrativen Nachfragens. „Narrative Interviews setzen zu ihrer Anwendung voraus, daß hinsichtlich des interessierenden Gegenstandsbereiches eine zentrale Eingangsfrage mit narrativer Generierungskraft formulierbar ist. Nur dort, wo ein Informant als Akteur oder Betroffener auf die Verstrickung in einen lebensgeschichtlichen oder historischen Ereigniszusammenhang (eine .Geschichte') zurückblicken kann, ist die Formulierung eines narrativen Themas möglich" (Schütze 1982, p. 570).

Die zentrale Anfangsthemenstellung hat die Funktion, eine längere Haupterzählung hervorzulocken. Die Eingangsfrage soll so gestaltet werden, daß der Forscher sein Interesse am gesamten Hintergrundzusammenhang bekundet, ohne jedoch eine allgemeine Erzählfolie in allen relevanten Aspekten vorzugeben. Gleichzeitig soll die Erzählung durch sie thematisch begrenzt werden. „Denn ansonsten kann das Problem auftauchen, daß der Erzähler den narrativen Zusammenhang (die Gesamtgestalt und den ,roten Faden" der historischen Ereignisse) nicht mehr überblickt ... Zudem muß der in der Erzählung darzustellende Ereigniszusammenhang zumindest in einem Hauptereignishöhepunkt. einem Ereignis zu Beginn und einem Ereignis gegen Ende des Ereignisablaufs noch im Gedächtnis des Erzählers haften, un überhaupt die Motivation fürs Erzählen über die vorgeschlagene Thematik zu schaffen" (Schütze 1982, p. 574). Während der Haupterzählung beschränkt sich der Interviewer ,strikt auf die Zuhörerrolle mit den entsprechenden erzählunterstützenden Signalen (Kopfnicken. .hm. hm usw.) bis der Informant durch eine eindeutige Koda... ıu erkennen gibt, daß nunmehr seine Erzählung abgeschlos- 
sen sei. Die jetzt einsetzenden Fragen des Interviewers dürfen nicht als Theorie-und Meinungsfragen formuliert sein (Fragen nach Gründen, Aufforderungen zu Meinungskundgaben usw.), sondern sollen neue narrative Sequenzen zu Darstellungsbereichen hervorlocken, die bisher nicht genügend oder überhaupt nicht ausgeführt wurden“ (Schütze 1982, p. 570). Um den Erzählungsfluß so wenig wie möglich zu stören und andererseits dem Interviewer die Möglichkeit zu geben, seine eigenen thematischen Interessen in die Interviewsituation einzubringen, sollen dabei zunächst durch „immanente“ Fragen Themenbereiche aufgegriffen werden, die der Informant selbst bereits angesprochen hat. Nur wenn der Informant bestimmte Punkte der Vorab-Erzählfolie gänzlich ausläßt, sollen auch „exmanente“ Fragen eingebracht werden.

Während Schützes Anweisungen - jedenfalls so weit - für die sozialwissenschaftliche Erzählforschung allgemeingültig sein dürften, reichen die Bestimmungen des narrativen Interviews jedoch nicht aus, um die Realitätshaltigkeit der Erhebungssituation für die Untersuchung von Lebensorientierungen zu gewährleisten. Wir werden mit dem partnerzentrierten Gespräch daher eine Methode vorschlagen, die dem narrativen Interview zwar verwandt ist, gleichzeitig jedoch wichtige Elemente der klientenzentrierten Gesprächstherapie (Rogers 1972) und der themenzentrierten Interaktion (Cohn 1976) übernimmt (vgl. Abb. 2).

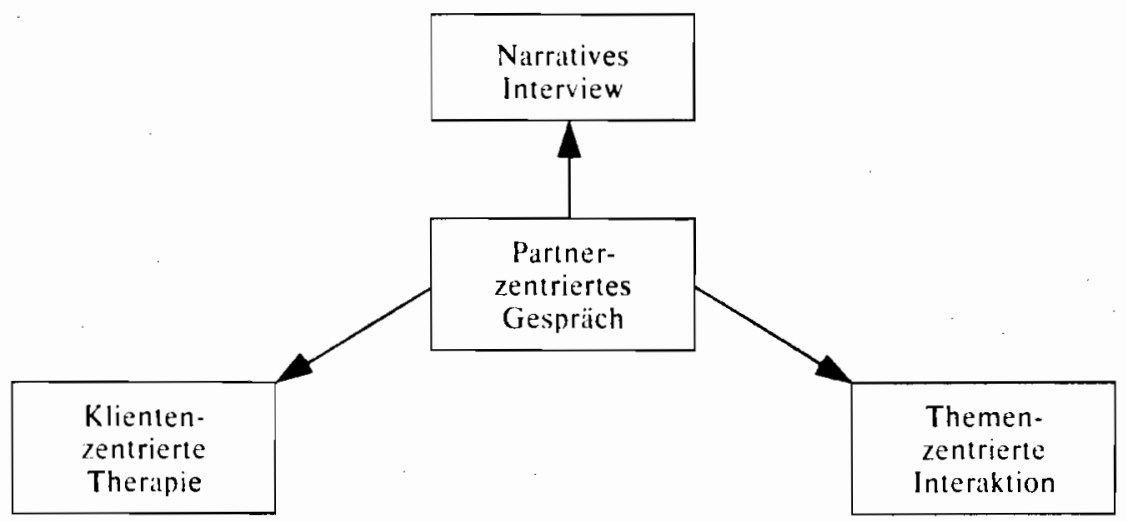

Abb. 2: Stellung des partnerzentrierten Gesprïchs zwischen narrativem Interview. klientenzentrierter Therapie und themenzentrierter Interaktion

Wie Hoffmann-Riem (1980, p. 347) zu Recht vermerkt hat. entscheidet sich mit dem Aufbau der Kommunikationsbeziehung, ,welche Tiefenschicht der Datengewinnung erreicht wird". Die Realitïtshaltigkeit einer Gesprächssituation in Hinblick auf die Lebensorien- 
tierungen eines Gesprächspartners setzt daher nicht nur voraus, daß über die Stimulation des Geschichtenerzählens auf die Gewinnung eines hinreichend kontexthaltigen Daten materials abgezielt wird. Die Realitätshaltigkeit kann erst gewährleistet werden, wenn der Gesprächspartner zugleich bereit ist, tatsächlich seine subjektive Realität der Dinge ein Stück weit offenzulegen und, wenn die Gesprächssituation wesentliche Strukturübereinstimmung mit typischen Alltagssituationen aufweist, die eben ein solches Offenlegen subjektiver Realität ermöglichen. Damit gewinnt die Art der Gesprächsbeziehungen zwischen dem Forscher und seinem Gesprächspartner besondere Bedeutung. Während Schütze den Gesprächspartner in erster Linie als „Informanten“ sehen kann, den es gilt, in bestimmte Zugzwänge des Erzählens (Detaillierungszwang, Gestaltschließungszwang, Kondensierungszwang) zu verwickeln, und die kommunikative Aufgabe des Interviewers im Eingehen der Rolle eines „produktiv zuhörenden Erzählpartners“ sieht, der seinem Gegenüber durch „erzählunterstützende Signale" sein Interesse an den Erzählungen signalisiert und seinen Einfluß auf den Interaktionsprozeß und die Entwicklung der Erzählung durch Einhalten einer Reihe von Verhaltensmaßregeln it auf ein Minimum beschränkt, wird der Forscher durch das partnerzentrierte Gespräch sowohl in seinem Selbsterfahrungsstand als auch in der Reflexion über sein Forschungsinteresse stärker gefordert.

Während Schütze für das narrative Interview davon ausgehen kann, daß die Fragen des Interviewers nicht als Eindringen in die private Sphäre erlebt werden und daher Sachverhalte treffen sollen, die auch von öffentlichem Interesse sind und von daher die Erzählung rechtfertigen, können wir uns bei der empirischen Analyse von Lebensorientierungen nicht von vorneherein nur auf Themen von öffentlichem Interesse beschränken. Durch die Spaltung in Inhalte „von öffentlichem Interesse" einerseits und „privaten“ Inhalten andererseits wird der Zugang zu Lebensorientierungen, welche ja den Gesamtzusammenhang individuellen Handelns und Erlebens - und damit auch die Verankerung des Öffentlichen im Privaten - herstellen, abgeschnitten. Für die empirische Erfassung von Lebensorientierungen bedarf es einer Bereitschaft zur Preisgabe subjektiver Realität, die auch die Preisgabe ,privater“ Realität - d. h. solcher Realität, die mensch nicht gleich jedermann zu erzählen, gleichsam zu veröffentlichen bereit ist - zumindest tendenziell mit einschließt. Die alltagsweltliche Kommunikationsform, an welche wir dabei anschließen können, ist die eines vertrauensvollen Gespräches zwischen einem Erzähler und einem Zuhörer, die ein gemeinsames Interesse an dem Gespräch miteinander verbindet. Dies stellt einerseits besondere Anforderungen an die Gesprächsführung und bedeutet andererseits, dal der Gespriichspartner nicht als "Informant" funktionalisiert werden kann, 
sondern daß der Forscher mit seinen Gesprächspartnern eine soziale Beziehung eingeht, die durch ein gemeinsames Interesse an dem Forschungsthema aber zugleich auch durch ein gegenseitiges Interesse von Forscher und Gesprächspartner gekennzeichnet sein muß. Die daraus sich ergebende Notwendigkeit der Überwindung der Distanz zwischen Forscher und Forschungssubjekt hat Smedslund sehr prägnant formuliert: „If you want to study a thing, you have to move closer. Create a situation of trust, show care, respect and understanding ". 17 Was dem Forscher dadurch abverlangt wird, ist mehr als bloß die Entwicklung bestimmter Gesprächstechniken, damit sich sein Gegenüber z. B. „nicht ausgefragt" fühlt. Es ist die Einnahme einer bestimmten Haltung gegenüber seinem Gesprächspartner, die nicht nur einen solchen Eindruck zu vermeiden hilft, sondern die von vorneherein ausschließt, daß der andere bloß zu Forschungszwecken funktionalisiert wird.

Die Vorstellung, daß das Interesse des Forschers an seinem Forschungsthema und sein Interesse an dem Gesprächspartner einander widersprechen, und daß so das Interesse am Forschungsthema notwendigerweise zur Funktionalisierung des Gesprächspartners führen muß, ist mit Sicherheit falsch. Jedenfalls dann. wenn sich das Interesse an dem Forschungsthema aus dem Interesse an den Menschen ergibt, aus dem Interesse, neue Erfahrungen mit Menschen zu machen, denen ich über ein gemeinsames Thema solidarisch verbunden bin, besteht ein solcher Widerspruch nicht. Solange das Interesse an dem Forschungsthema dagegen hauptsächlich durch z. B. akademische Qualifikationswünsche geprägt ist, ist der Widerspruch unauflösbar. Die Tencienz, andere Menschen (oft sogar in den intimsten Zweierbeziehungen) zu funktionalisieren, ist uns durch unsere Sozialisation so tief eingeprägt, daß sie nicht einfach per Beschluß abgelegt, ja ohne entsprechende Selbsterfahrungsprozesse nicht einmal in verständlicher Weise über die Möglichkeit ihrer Überwindung kommuniziert werden kann. Es ist daher auch müßig, hierüber weiter theoretisieren zu wollen.

Wenn wir von der Erwartung ausgehen, daß unsere Gesprïchspartner uns Vertrauen und Offenheit entgegenbringen wollen, dann müssen wir ihnen auch von unserer Seite Vertrauen und Offenheit entgegenbringen. Dazu gehört auch die Offenlegung des Interesses, welches der Forscher an dem Gespräch hat, denn nur so kann sich ein gemeinsames Interesse an dem Gespräch konstituieren. Und es gehört dazu. daß die Rollenverteilung während des Gesprächs klargemacht wird, dal3 das Interesse des Forschers ein Hörinteresse und das seines Gesprächspartners ein Erzählinteresse ist. Eine sich unter der Fiktion egalitärer Kommunikation (Hoffmann-Riem 1980, p. 380) allzuleicht einstellende Rollenverwischung birgt die Gelahr in sich, dab der 
Gesprächspartner Zweifel an der Offenheit des Forschers entwickelt. Darüber gewinnen die Kontaktaufnahme mit dem Gesprächspartner und die dem eigentlichen Gespräch vorangehende „small-talk-Phase“ (Schütze) besondere Bedeutung. Sie sind nicht nur entscheidend für die Herstellung des Rapports zwischen dem Forscher und seinem Gesprächspartner, sondern müssen auch für die Konstitution eines gemeinsamen Interesses an dem Gespräch und für die Klärung der während des Gesprächs eingegangenen Rollenverteilung genutzt werden. Deshalb ist auch die Trennung zwischen dem vorangehenden „small-talk“ und der das eigentliche Gespräch eröffnenden Eingangsfrage eher künstlich. Die Eingangsfrage hat lediglich die Funktion, das Gesprächsthema nochmals auf den Punkt zu bringen. Die Bedeutung der Eingangsfrage wird durch den Kontext der bisherigen Kommunikation zwischen dem Forscher und seinem Gesprächspartner mitbestimmt und kann von diesem nicht getrennt werden. Alle quasiobjektivistischen Vorstellungen, die Eingangsfrage könnte als ,narrativer Stimulus"standardisiert - und die sich anschließende Erzählung könnte als „narrative Reaktion“ auf diesen Stimulus betrachtet werden, sind Restbestände einer im traditionellen Paradigma verhafteten Denkweise, die zwar nicht apodiktisch abgelehnt werden kann, gleichwohl jedoch ungeeignet ist, um die methodische Vorgehensweise interpretativer Sozialforschung angemessen $z u$ thematisieren. Die Notwendigkeit der Formulierung einer - allerdings flexiblen - Folie für die - ggf. kontextabhängig zu modifizierende - Eingangsfrage bleibt davon jedoch unberührt.

Der empirische Zugang zu den Lebensorientierungen von Menschen bleibt uns verwehrt, wenn unsere Gesprächspartner nicht bereit sind, ihre subjektive Realität der Dinge offenzulegen. Um ihnen dies zu ermöglichen, ist es erforderlich, daß der Forscher während des gesamten Gesprächs jene personenzentrierte Grundhaltung einnimmt und auch kommuniziert, die Rogers (1972) unter den Kategorien „einfühlendes Verstehen“, „Wertschätzung und emotionale Wärme“ sowie "Echtheit und Selbstkongruenz" thematisiert. Daraus lassen sich eine Reihe von Regeln ableiten, welche für die Gesprächsführung zu beachten sind, und die u.a. Tausch \& Tausch (1981) ausführlich diskutieren und in eine lehrbare Form zu übersetzen versuchen. Tausch und Tausch weisen auch darauf hin, daß diese Regeln nicht als Rezepte verstanden werden können, nach denen eine personenzentrierte Haltung mechanisch „verwirklicht" werden könnte.

Ich möchte auf Rogers Kategorien hier nur so weit eingehen, als es zur Abgrenzung des partnerzentrierten Gesprächs gegenüber dem narrativen Interview erforderlich ist, bzw. soweit das partnerzentrierte Gespräch als Forschungsmethode zusätzliche Konkretisierungen der Kategorien erfordert. Der empirische Zugang zu den Lebens- 
orientierungen von Menschen setzt voraus, daß wir uns einerseits unter Einnahme einer "Innenperspektive“ in den subjektiven Bezugsrahmen unserer Gesprächspartner hineinversetzen, daß wir versuchen, die Dinge seiner Bedeutungswelt so wahrzunehmen und zu erleben, wie er sie wahrnimmt und erlebt, und versuchen, seine Haltung, seine subjektiven Erfahrungen, Werte und Wahrnehmungen zu akzeptieren und anzunehmen. Andererseits haben wir uns unter Einnahme einer ,äußeren Perspektive“ aus dem subjektiven Bezugsrahmen unseres Gegenübers wieder zu lösen und jene Orientierungen kritisch zu hinterfragen, vermittels welcher diese subjektive Realität sich konstituiert. Dieser Perspektivenwechsel betrifft zunächst nur den Interpretationsprozeß und nicht die Gesprächsführung, die allerdings dafür ausschlaggebend ist, ob wir uns denn überhaupt die Möglichkeit eröffnen, das dafür erforderliche empirische Material zu erlangen.

Um den subjektiven Bezugsrahmen unseres Gesprächspartners übernehmen zu können, ist es erforderlich, daß er uns tatsächlich erzählt, was er erlebt (hat), und nicht nur seine - bereits durch Rationalisierungen, Abstraktionen und Generalisierungen etc. bereinigten Alltagstheorien darüber. Dies hat Konsequenzen für die Art der Formulierung der Fragen im partnerzentrierten Gespräch, die Smedslund in die Regel zusammengefaßt hat: "Don "t ask what goes on inside people, but ask what people go on inside of".18 Diese Regel geht parallel zu der von Schütze geforderten Vermeidung von Theorie-, Meinungs- und „warum"-Fragen im narrativen Interview, geht aber zugleich darüber hinaus, indem sie einen Weg aufzeigt, wie wir stattdessen fragen können, z. B. indem wir ,warum “-Fragen durch „wieso"Fragen ersetzen. Statt unseren Gesprächspartner um Erklärung zu bitten, warum er diese oder jene Handlung ausgeführt, sich diese oder jene Meinung gebildet hat etc., können wir ihn danach fragen, wie es sich so ergeben hat, daß er dies getan, wie er dazu gekommen ist, oder wir können ihn auch zu solchen Erläuterungen bewegen, ohne explizit danach zu fragen, indem wir z. B. die Inhalte seiner Meinungsäußerungen mit einem leicht fragenden Anheben der Stimme am Satzende .spiegeln“ und ihn derart implizit auffordern, sie mit mehr Kontext zu versehen. $19 \mathrm{Um}$ die Bereitschaft zur Preisgabe subjektiver Realität zu schaffen bzw. nicht zu zerstören, ist es darüber hinaus erforderlich, daß der Forscher bereit ist, die subjektive Realität seines Gegenübers als solche zu akzeptieren, den Gesprächspartner nicht etwa in Diskussionen verwickelt und sich mit eigenen Stellungnahmen zurückhält. Das heißt auch, daß er sich der Subjektivität seiner eigenen Realität bewußt ist.

Dies bedeutet nicht, daß der Forscher - wie dies im narrativen Interview gefordert wird - seinem Gesprächspartner zu signalisieren 
hat, daß er dessen Typisierungen in einer Weise nachvollzieht, ,als ob beim Austausch der Standorte , der Zuhörer als Erzähler die Dinge' in derselben Typikalität sähe" (Hoffmann-Riem 1980, p. 359) sondern es bedeutet, daß der Forscher seinem Gegenüber zu signalisieren hat, daß es in dem Gespräch nicht um irgendeine „objektive“ Realität geht, über die sich der Forscher und sein Gesprächspartner im Verlauf des Gesprächs zu einigen hätten, sondern daß lediglich die Art und Weise, wie der Gesprächspartner die Dinge sieht, von Interesse ist, daß der Forscher bereit ist, sich auf die subjektive Realität seines Gegenübers voll und ganz einzulassen. Um dies zu ermöglichen, ist es erforderlich, daß sich der Forscher schon während des Gesprächs so weit als möglich in den subjektiven Bezugsrahmen seines Gegenübers hineinversetzt, die Übernahme des Bezugsrahmens im Sinne der Regeln für die Gesprächsführung in der klientenzentrierten Therapie kommuniziert und seine Fragen nicht nur in dem Sinne „immanent" formuliert hat, als sie Themen aufgreifen, die sein Gesprächspartner bereits angesprochen hat, sondern indem er die Fragen aus dem subjektiven Bezugsrahmen des Gesprächspartners heraus entwickelt. Dabei darf man sich allerdings nicht allzu großen Illusionen darüber hingeben, wie weit die Übernahme des Bezugsrahmens des Gesprächspartners schon während des Gesprächs de facto gelingen kann. Es erfordert systematische Anstrengungen, jene Mechanismen des Alltagsbewußtseins zu durchkreuzen, die das Neue, welches unser Gegenüber uns erzählt (hat), vorschnell auf das - aus eigener Erfahrung, unserem theoretischen Wissen, vorangegangenen Gesprächen oder unserer sonstigen Kenntnis der Sachlage - vermeintlich Bekannte zu reduzieren. Und, die Bereitschaft, die subjektive Realität eines anderen - auch wenn man sie im Moment noch nicht ganz versteht - anzuerkennen und nachzuvollziehen, erfordert darüber hinaus eine Menge an Selbsterfahrung darüber, welchen eigenen Bedürfnissen und Ängsten diese Mechanismen entgegenkommen, aus welchen Widerständen sie geboren sind, welche Bedrohlichkeit die subjektive Realität des anderen für unser eigenes Selbstverständnis hat. ${ }^{20}$

Für das partnerzentrierte Gespräch ergibt sich daraus, daß mit direkten Fragen vorsichtig umgegangen werden und soweit als möglich auf die Methode des Spiegelns ausgewichen werden sollte. Wo direkte Fragen unvermeidlich sind, kann durch gezielte Ausnützung der „eigentümlichen Vagheit" der Alltagskommunikation vermieden werden, daß sich der Forscher definitiv außerhalb des Bezugsrahmens seines Gesprächspartners stellt. Jedenfalls sollten ad-hoc-Interpretationen des Gehörten - etwa indem man es „auf den Punkt zu bringen" oder zusammenzufassen versucht - vermieden werden. Stattdessen sollte der Farscher versuchen, dem Erzählfluß zu folgen und 
seine Aufmerksamkeit voll und ganz auf das hier und jetzt Gesagte auszurichten, ohne schon während des Gesprächs den Gesamtzusammenhang kognitiv erfassen zu wollen. Indem wir den Erzählfluß unseres Gesprächspartners nicht durch (voreilige) Fragen unterbrechen, (vermeintlichen) Themenabweichungen folgen und Pausen aushalten, vermitteln wir ihm bezüglich der Form seiner Erzählung zugleich bereits jene „nicht an Bedingungen gebundene positive Wertschätzung" (Tausch \& Tausch 1981, p. 70), die wir ihm auch bezüglich der Inhalie seiner Erzählung entgegenbringen müssen - d.h. ohne Abneigung oder Mißbilligung auszudrücken -, wollen wir die Bereitschaft zur ungeschminkten Darstellung seiner subjektiven Realität nicht im Keim ersticken.

Die Forderung nach Vermittlung einer nicht an Bedingungen geknüpften positiven Wertschätzung wird durch die Unterdrückung von Kritik freilich bei weitem noch nicht erfüllt. Tausch \& Tausch (1981, p. 71) weisen darauf hin, daß auch mechanische Formulierungen oder mangeIndes Interesse an dem Gesagten ein Defizit an positiver Wertschätzung ausdrücken. Eben diese Gesprächshaltungen entstehen aber oft gerade dann, wenn z.B. die Äußerung von Kritik unterdrückt wird. Jede Art von „Selbstdisziplinierung“ des Forschers stört die kommunikative Beziehung mit seinem Gegenüber. Aus diesem Grunde ist der Ech theit und Selbstkongruenz des Forschers der unbedingte Vorrang vor den anderen beiden Kategorien einzuräumen. welche eine personenzentrierte Gesprächshaltung ausmachen. Dies bedeutet, daß Störungen nicht unterdrückt werden dürfen. Der Forscher darf nicht versuchen, „sich nichts anmerken zu lassen“, sondern muß - wie dies auch in der themenzentrierten Interaktion gefordert ist - die Störung in geeigneter Weise thematisieren. In geeigneter Weise, d.h. indem er - ohne Vorwürfe gegen seinen Gesprächspartner zu erheben - diesem mitteilt, welche Wirkungen das Gesagte - oder das sonstige Verhalten des Gesprächspartners - auf ihn hat. Ob eine solche angemessene Thematisierung von Störungen gelingen kann, hängt allerdings weniger von erlernbaren Techniken ab, als davon, ob wir unserer selbst genügend sicher sind, im Konfliktfall auf unser Gegenüber einen Schritt zugehen zu können (indem wir ihm etwas über uns mitteilen), statt uns von ihm zu distanzieren (indem wir Wertungen seiner Person oder seines Verhaltens abgeben). Diese Sicherheit kann im Grunde nur über Selbsterfahrungsprozesse erworben und durch regelmäßige Supervision aufrechterhalten werden.

Wo diese Voraussetzungen fehlen und wir eine personenzentrierte Gesprächshaltung per Regelbefolgung schematisch herzustellen versuchen, verlieren wir den Anschluß an die natürliche Alltagskommunikation. verfallen in ein "therapeutisches Gehabe“. und die Realitätshaltigkeit des Gesprächs wird zerstört. Aus diesem Grunde sind dic 
oben formulierten Regeln nicht als Verhaltensinstruktionen für die Gesprächsführung zu verstehen, sondern als ein System von Regeln, anhand dessen wir unser kommunikatives Verhalten reflektieren und auf seine Eignung für die Gewinnung jener Art von empirischem Material beurteilen können, welches für die deskriptive Erfassung individueller Subjektivität erforderlich ist.

\section{Anmerkungen}

1 Vgl. dazu z. B. das „Lehrbuch der Psychologie“ von Ruch \& Zimbardo (1974), das gleich auf Seite 12 dogmatisch festhält: „Die Ausgangsposition eines jeden Wissenschaftlers ist die Annahme eines auf GesetzmäBigkeiten beruhenden Universums".

2 Mit dem Wort "Dinge" werden hier nicht nur die belebten und unbelebten Gegenstände der Natur gemeint, sondern auch abstrakte Gegenstände wie z. B. Gesellschaft. Institutionen, Ideen, Handlungen anderer Personen usw.. Schlichtweg alles. was mensch in seiner Welt wahrzunehmen und/oder worauf er sein Handeln auszurichten vermag.

3 Weder das Wort „Ansichten“, noch das Wort „Bedeutung“ soll dabei allzu rationalistisch verstanden werden.

4 Vgl. dazu die Ausführungen zum Verhältnis von "Wahrheit“ und „Wirklichkeit“ in Kamlah \& Lorenzen (1967) sowie bei Janich, Kambartel \& Mittelstrass (1974).

5 Holzkamp (1972) hat darauf aufmerksam gemacht, daß sich die Kriterien des Bestätigungsgrades empirischer Hypothesen und der Strukturähnlichkeit zwischen Forschungssituation und Anwendungssituation im traditionellen Paradigma gegenseitig ausspielen. Andererseits kann technische Relevanz psychologischer Forschung nur erzielt werden, wenn beide Kriterien gleichzeitig erfüllt sind.

6 Die hier verwendeten logischen Symbole bezeichnen: " $\wedge$ " den Allquantor $(\wedge x$ $=$ "für alle $\left.x^{\prime \prime}\right)$ und ., "die Subjunktion $(A \rightarrow B=$, wenn $A$, dann $B "$ "), .A " die Konjunktion $(A \wedge B=, A$ und $B$ ) und , $>$ “ die logische lmplikation $(A>B=$,aus A folgt B").

$7 \mathrm{Vgl}$. dazu die Kritik Fromms (1949) an Kardiner, die u. a. eben darauf hinausläuft, daß die sozio-ökonomischen Faktoren bei Kardiner (ähnlich wie bei Adorno) nur als Randbedingungen der Persönlichkeitsentwicklung aufscheinen. An anderer Stelle weist Fromm (1932, p. 41) ausdrücklich darauf hin, daß Freud niemals den isolierten, aus dem sozialen Zusammenhang gelösten Menschen als Objekt der Psychologie angenommen hat. ..Ist so für Freud immer nur der vergesellschaftete Mensch, der Mensch in seiner sozialen Verflochtenheit, Objekt der Psychologie, so spielen auch für ihn ... Umweit und Lebensbedingungen des Menschen die entscheidende Rolle für seine seelische Entwicklung wie für deren theoretisches Verständnis“. Darauf aufbauend kritisiert Fromm an der Anwendung der Psychoanalyse auf gesellschaft liche Probleme, daß es ein Fehler der psychoanalytischen Autoren gewesen sei, die psychoanalytische Methode nicht mehr "in konsequenter und korrekter Weise anzuwenden, wenn sie statt über Individuen über Gesellschaften, Gruppen, Klassen, kurz über soziale Phänomene Untersuchungen anstellten“ (p. 45): „Man sah zwar. daß der einzeine nur als vergesellschaftetes Wesen zu verstehen ist, man entdeckte. daß es die Beziehungen des Kindes zu den verschiedenen Mitgliedern der Familie sind, die seine Triebentwicklung so entscheidend bestimmen, aber man übersah fast volkommen, daß die Familie ihrerseits in ihrer ganzen psychologischen und sozialen Struktur, mit den für sie spezifischen Erziehungszielen und affektiven Einstellungen. das Produkt einer bestimmten gesellschaftlichen und. im engeren Sinn. einer besimmten Klussenstruktur ist, daß sie tatsächlich nur die psychologische 
Agentur der Gesellschaft und Klasse ist, aus der sie erwächst" (p. 43). In seinen eigenen empirischen Untersuchungen zum Gesellschaftscharakter (Fromm \& Maccoby 1970. Fromm 1980) bediente sich Fromm daher - anders als Adorno - hauptsächlich des offenen Fragebogens, aber z. T. auch schon des Interviews (vgl. Fromm 1936). Die Antworten wurden nicht „mechanisch“ ausgewertet, sondern unter Bezugnahme auf Fromms Charaktertypen und im Kontext der sozioökonomischen Situation der Befragten psychoanalytisch interpretiert.

${ }^{8}$ Im Gesellschaftscharakter sind jene, von den Mitgliedern einer Gesellschaft gemeinsam geteilten Selbstverständlichkeiten ihres Erlebens und Handelns niedergelegt, aufgrund derer es eben keiner bewußten Entscheidung mehr bedarf, ob sie sich an das gesellschaftliche Modell halten wollen oder nicht.

9 Dabei sind die von Habermas geleisteten Vorarbeiten zu einer Konsenstheorie auf die Konstruktion des hermeneutischen Feldes II zu applizieren: „Die Unterscheidung des wahren und des falschen Konsensus muß in Zweifelsfällen durch Diskurs entschieden werden. Aber der Ausgang des Diskurses ist wiederum von der Erzielung eines tragfahigen Konsensus abhängig. Die Konsensustheorie der Wahrheit bringt zu Bewußtsein, daß über die Wahrheit von Aussagen nicht ohne Bezugnahme auf die Kompetenz möglicher Beurteiler, und über diese Kompetenz wiederum nicht ohne Bewertung der Wahrhaftigkeit ihrer Äußerungen, der Richtigkeit ihrer Handlungen entschieden werden kann" (Habermas 1971, p. 134).

Leithäuser \& Volmerg (1977, p. 133) weisen darauf hin, daß die Forschergruppen des hermeneutischen Feldes Il immer wieder in diesen Zirkel geraten werden. Er ist als philosophisches Problem nicht auflösbar; andererseits kann er. bezogen auf die empirischen Interpretationen, nicht so einschränkend und rigide formuliert werden, daß 3 dadurch ausreichende Plausibilität der Interpretationen ... von vorneherein ausgeschlossen wäre".

11) Die Arbeitsgruppe Bielefelder Soziologen spricht hier von „Rollenübernahme“. Ich selbst werde weiter unten in Anlehnung an Rogers von der "Übernahme des subjektiven Bezugsrahmens" eines Gesprächspartners sprechen. Beide Begriffe sind jedoch enger gefaß $3 t$ als der Begriff der „praktischen Teilhabe“ und in ihrer Anwendbarkeit an bestimmte Methoden gebunden. So weisen Leithäuser \& Volmerg (1977. p. 134) darauf hin, daß der Begriff der Rollenübernahme zwar etwas für die Methode der „teilnehmenden Beobachtung" zutreffen mag, nicht immer aber für die Methode der ..themenzentrierten Interaktion" und, wie Lorenzer gezeigt hat, auch nicht für das psychoanalytische Verfahren.

1 Holzkamp (1970) hat darauf hingewiesen und davor gewarnt, daß die Strukturübereinstimmung zwischen Erhebungssituation und Anwendungssituation - und damit die technische Relevanz experinentalpsychologischer Forschung - auch aul umgekehrtem Weg hergestellt werden kann: indem man die Struktur der Alltagsrealität in höherem Maße der Struktur der experinentellen Realität angleicht

12 Als dritte Möglichkeit der Handlungsbeschreibung sei hier auf die Beschreibung einer Handlung durch die Unterstellung bestimmter Handlungsorientierungen verwiesen.

13 Kamlah \& Lorenzen (1967) sprechen in diesem Zusammenhang von der „Aktualisierung eines Handlungsschemas".

it Auch hierin kommt wieder das Bestreben des Alltagsbewußtseins zum Ausdruck. beim Vertrauten zu bleiben, das (eventuell) Neue in der sozialen Situation auf das Bekannte, aber nur vermeintlich und vage Bekannte, zu reduzieren. Daßs die Struk. tur unserer Sprache solche Möglichkeiten eröffnet, stellt zugleich die Grundlage für den in der traditionellen empirischen Sozialforschung verbreiteten Glauben dar. Handlungen könnten .operational" beschrieben werden - womit dann jene Sinn. deutungen, die ein beobachibares Verhalten erst zur Handlung nachen, lediglich unreflektiert hleiben und als unproblematisch unterstellt werden. 
15 Die Feststellung der Bedeutung von Handlungen und der diese Bedeutungen konstituierenden Lebensorientierungen können allerdings nicht in aufeinanderfolgende methodische Schritte aufgelöst werden, sondern sie bewegen sich in einem hermeneutischen Zirkel.

16 Darunter 2. B. das Verbot von Theorie-, Meinungs- und „warum“-Fragen. Weiter sollte der Interviewer nicht zu erkennen geben, wenn ihm bestimmte Teile der Erzählung bereits bekannt sind, Auslassungen sollten nicht explizit nachgefragt werden etc.

17 Diskussionsbeitrag aüf der Arbeitstagung „Erfahrung und Struktur in der psychologischen Forschung" am Wissenschaftskolleg zu Berlin, 28.-30. Juni 1984.

18 Diskussionsbeitrag auf der o.a. Arbeitstagung.

19 Das Verbot von Theorie-, Meinungs- und „warum"-Fragen ist dabei lediglich in Hinblick auf die für die empirische Erfassung von Lebensorientierungen erforderliche Datenqualität begründet und nicht apodiktisch zu verstehen. Dasselbe gilt auch für die Zurückhaltung des Forschers mit eigenen Meinungsäußerungen und für die Regel, wonach der Gesprächspartner nicht in Diskussionen verwickelt werden soll. Je mehr wir diese Regeln verletzen, desto mehr nähert sich die Gesprächssituation der themenzentrierten Interaktion an. Die Realitätshaltigkeit des empirischen Materials verschiebt sich dann zugunsten der Übertragungsprozesse des Alttagsbewußtseins.

20 Der Ort, an dem diese systematischen Anstrengungen zu erfolgen haben. ist der Interpretationsprozeß, der sich dadurch grundlegend von der „Verständnisbildung“ in der Alltagskommunikation unterscheidet, die zu einem großen Teil nicht-hermeneutisch. sondern durch Abwehr und Übertragung bestimmt ist. Aus diesem Grunde sind auch Bedenken gegenüber Interpretationsverfahren anzumelden, die wie z. B. Südmersen (1983) - den hermeneutischen Prozeß aus Gründen der Arbeitsökonomie abzukürzen versuchen. Ich teile die von Heinze \& Klusemann (1980, p. 109) vertretene Auffassung, wonach sich eine einzelne Textstelle in ihrer wirkiichen Bedeutung erst erschließt, wenn der Gesamtkontext bekannt ist. „Der Interpretationsvorgang bedeutet also ein ständiges Schließen vom Ganzen auf das Einzelne und umgekehrt". Die von Südmersen vorgeschlagene Gliederung des Textes in Sequenzen ist dabei durchaus funktional, um einen .engeren“ Kontext der Textstelle herzustellen, der seine Bedeutung wiederum erst aus dem Kontext des Gesamtinterviews gewinnt. Die Notwendigkeit einer gründlichen, Satz für Satz vorgehenden Textinterpretation kann jedoch nicht behoben werden.

\section{Literatur}

adorno, T. W. Frenkel-Brunswik, E., Levinson, D. J. \& Sanford, R. N. 1950. The authoritarian personality. New York: Harper \& Row.

Arbeitsgruppe Bielefelder Soziologen. 1978. Kommunikative Sozialforschung. München: Fink.

ARgelander. H. 1972. Der Flieger. Frankfurt a. M.: Suhrkamp.

Aschenbach. G. 1981. Plädoyer für eine Psychologie des "reflexiven Subjektes“. Zur Kontroverse um Erklären und Verstehen. In: KemPF. W.\& AsChENBACH, G. (Ed.) Konflikt und Konfliktbewältigung. Bern: Huber.

ASCHENBACH, G. 1982. Konflikt- und Aggressionsbewältigung aus kulturwissenschaftlicher Sicht. In: Hilke, R. \& KempF, W. (Ed.) Aggression. Bern: Huber.

BriRgFr. P. \& LUCKMANN. T. 1969. Die gesellschaftliche Konstruktion der Wirklichkeit. Frankfurt a. M.: Fischer.

Bul Mt. H. H. 1973. Der methodologische Standort des symbolischen Interaktionismus. In: Arbeitscirl ppe Biflefelder Soziologen (Ed.) Alltagswissen. In teraktion und gesellschaftliche Wirklichkeit. Band 1. Reinbek: Rowohlt. 
Brandtstädter, J. 1981. Begriffliche Voraussetzungen der Moralpsychologie. In: KempF, W. \& Aschenbach, G. (Ed.) Konflikt und Konfliktbewältigung. Bern: Huber.

Buss, A. H. 1961. The psychology of aggression. New York: Wiley.

Cohn, R. C. 1976. Von der Psychoanalyse zur themenzentrierten Interaktion. Stuttgart: Klett.

Dollard, J., Doob, L. W., Miller, N. E., Mowrer, O. H. \& Sears, R. R. 1939. Frustration and aggression. New Haven: Yale University Press.

Fromm, E. 1932. Über Methode und Aufgabe einer analytischen Sozialpsychologie. In: Fromm, E. Gesamtausgabe. Band 1. Stuttgart: Deutsche Verlags-Anstalt, 1980.

Fromm, E. 1936. Autorität und Familie. Geschichte und Methoden der Erhebungen. In: Fromm, E. Gesamtausgabe. Band 3. Stuttgart: Deutsche Verlags-Anstalt, 1981.

Fromm, E. 1949. Über psychoanalytische Charakterkunde und ihre Anwendung zum Verständnis der Kultur. In: From , E. Gesamtausgabe. Band 1. Stuttgart: Deutsche Verlags-Anstalt, 1980.

Fromm, E. 1962. Jenseits der Illusionen. Die Bedeutung von Marx und Freud. In Fromm, E. Gesamtausgabe. Band 9. Stuttgart: Deutsche Verlags-Anstalt, 1981.

Fromm, E. 1980. Arbeiter und Angestellte am Vorabend des Dritten Reiches. Eine sozialpsychologische Untersuchung. In: Fromm, E. Gesamtausgabe. Band 3. Stuttgart: Deutsche Verlags-Anstalt, 1981

Fromm. E. \& Maсcoby, M. 1970. Psychoanalytische Charakterologie in Theorie und Praxis. Der Gesellschaftscharakter eines mexikanischen Dorles. In: Fromm, E. Gesamtausgabe. Band 3. Stuttgart: Deutsche Verlags-Anstalt, 1981.

GaRfinkel, H. 1973. Das Alltagswissen über soziale und innnerhalb sozialer Strukturen. In: Arbeitsgruppe Bielefelder Sozlologen (Ed.) Alltagswissen, Interaktion und gesellschaftliche Wirklichkeit. Band 1. Reinbek: Rowohlt.

Garfinkel. H. \& SACKS, H. 1973. Anhang: Zum Phänomen der Indexikalität. In Arbeitsgrtple. Biflefelder Soziologen (Ed.) Alltagswissen, Interaktion und gesellschaltliche Wirklichkeit. Band 1. Reinbek: Rowohlt.

HablRmas, J. 1971. Vorbereitende Bemerkungen zu einer Theorie der konımunikativen Kompetenz. In: HABERMAS. J.\& LuHManN. N. (Ed.) Theorie der Gesellschaft oder Sozialtechnologie. Franklurt a. M.: Suhrkamp.

HEINZE. T. \& KLISEMANN. H. W. 1980. Versuch einer sozialwissenschaftichen Paraphrasierung am Beispiel des Ausschnittes einer Bildungsgeschichte. In HeINZF, T. KLlisfmav., H. W. \& SöfFNFR, H. G. (Ed.) Interpretationen einer Bildungsgeschichte. Überlegungen zur sozialwissenschattlichen Hernieneutik. Bensheim: päd. extra buchverlag.

Henlet, C. G. 1965. Aspects of scientific explanation and other essay's in the philosophy of science. New York: Free Press

Hit.kF, R. 1982. Das Problem generalisierender Behauptungen über die Aggressivität von Menschen als Problen der Reduktion von Qualität auf Quantität. In: HILKE, R. \& KemPF, W. (Ed.) Aggression. Bern: Huber.

Hilke, R, \& Kempf. W. 1976. Zur Rechtfertigung der Aggressionsmaschine. Zeitsehrift für Sozialpsychologie 7, 47-58.

HofFMavi-Rikm, C. 1980. Die Sozialforschung einer interpretativen Soziologie. Kölner Zeitschrift für Soziologie und Sozialpsychologie 32, 339-373.

Holzkamp. K. 1972. Zum Problem der Relevanz psychologischer Forschung für die Praxis. In: Holzkamp. K. Kritische Psychologie. Frankfurl a. M.: Fischer.

Jancil, P., Kambartel. F. \& Mittflstrass, J. 1974. Wissenschaftstheorie als Wissenichalftskritik. Frankfurt a. M.: Aspekte Verlag. 
Kambartel, F. 1978. Universalität als Lebensform - Zu den (unlösbaren) Schwierigkeiten, das gute und vernünftige Leben über formale Kriterien zu bestimmen. In: Oelmüller, W. (Ed.) Materialien zur Normendiskussion: Normenbegründung - Normendurchsetzung. Paderborn: Schöningh.

KAMBARTEL, F. 1981. Friede und Konfliktbewältigung, handlungstheoretisch und ethisch betrachtet. In: KempF, W. \& AschenbaCH, G. (Ed.) Konflikt und Konfliktbewältigung. Bern: Huber.

Kamlah, W. \& Lorenzen, P. 1967. Logische Propädeutik. Mannheim: Bibliographisches Institut.

KEMPF, W. 1978. Konfliktlösung und Aggression. Bern: Huber.

Kempf, W. 1981. Soziales Lernen. In: Werbik, H. \& KaISER, H. J. (Ed.) Kritische Stichwörter Sozialpsychologie. München: Fink.

KEMPF, W. 1982a. Mythen der Intelligenzforschung. Unterricht Biologie 6/82-73, 43-48.

KEMPF, W. 1982b. Soziale Motivation und Persönlichkeitsentwicklung - zur Sozialpsychologie der Konsum- und Wettbewerbsgesellschaft. Zeitschrift für Sozialpsychologie und Gruppendynamik 7, 3-15.

KEMPF, W. 1983a. Some theoretical concerns about applying latent trait models in educational testing. [n: Anderson, S. B. \& Helmick, J. S. (Ed.) On educational testing. San Francisco: Jossey-Bass.

KEMPF, W. 1983b. Korrelation, Argumentation und Reflexion - methodologische und sozialpsychologische Gedankensplitter zu drei Ebenen humanwissenschaftlicher Empirie. Zeitschrift für Sozialpsychologie und Gruppendynamik 8, 11-17.

KEMPF, W. 1984. Zur Sozialpsychologie der Friedensbewegung. Zeitschrift für Sozialpsychologie und Gruppendynamik 9, 26-37.

KEMPF, W. 1985. Aggressionsbewältigung und Friedenssehnsucht - Orientierungsleistungen und politisch-kultureller Wandel in der Friedensbewegung. In: SOMMER, G. \& Börner, A. (Ed.) Kriegsgefahr und Friedensengagement. Tübingen: Deutsche Gesellschaft für Verhaltenstherapie.

LANDA, L. N. 1969. Algorithmierung im Unterricht. Berlin (DDR): VEB Volk und Wissen.

Leithäuser. T. 1978. Formen des Alltagsbewußtseins. Frankfurt a. M.: Canıpus.

LeITHAllser. T. \& VOLMERG, B. 1977. Die Entwicklung einer empirischen Forschungsperspektive aus der Theorie des Alltagsbewußtseins. In: LeITHÄUSER. T.. Volmerg, B., Salje, G., Volmerg, U.\& Wutka. B. Entwurf zu einer Empirie des Alltagsbewußtseins. Frankfurt a. M.: Suhrkamp.

Leithäuser, T.. Volmerg, B., Salje, G., Volmerg, U. \& Wutka, B. 1977. Entwurf zu einer Empirie des Alltagsbewußtseins. Frankfurt a. M.: Suhrkamp.

LOREvZER. A. 1974. Die Wahrheit der psychoanalytischen Erkenntnis. Ein historisch-materialistischer Entwurf. Frankfurt a.M.: Suhrkamp.

Mertevs, W. 1975. Sozialpsychologie des Experiments. Hamburg: Hoffmann \& Campe.

Miller. N. E. 1941. The frustration-aggression hypothesis. In: Berkowitz, L. (Ed.) Roots of aggression. New York: Atherton. 1969.

Rocitrs, C. R. 1972. Die klientenzentrierte Gesprächspsychotherapie. München: Kindler.

Ricu. F. L. \& Zimaardo. P. G. 1974. Lehrbuch der Psychologie. Berlin: Springer. Sartrt, J. P. 1956. Das Sein und das Nichts. Reinbek: Rowohlt.

Scavol RA, J. M. 1973. Structural learning. Vol. I. Theory and research. New York: Gordon \& Breach.

SCANdTR I. J. M. \& Brainerd. C. J. (Ed.) 1978. Structural/Process models of complex human behavior. Alphen an den Rijn: Sijthoff \& Noordholf. 
Schütz, A. \& LuckmanN, T. 1979. Strukturen der Lebenswelt. Band I. Frankfurt a. M: Suhrkamp.

SCHÜTZE, F. 1976. Zur Hervorlockung und Analyse von Erzählungen thematisch relevanter Geschichten im Rahmen soziologischer Feldforschung - dargestellt an einem Projekt zur Erforschung kommunaler Machtstrukturen. In: ArbeitsgRUPPE Bielefelder Sozıologen (Ed.) Kommunikative Sozialforschung. München: Fink.

Schütze, F. 1982. Narrative Repräsentation kollektiver Schicksalsbetroflenheit. In: LÄMmERT, C. (Ed.) Erzählforschung: ein Symposium. Stuttgart: Metzler.

Schütze, F. 1983. Biographieforschung und narratives Interview. Neue Praxis 13. 283-293.

Schwemmer, O. 1976. Theorie der rationalen Erklärung. München: Beck.

Sears, R. R. 1941. Nonaggressive reactions to frustration. Psychological Review $48,343-348$

SMEDSLLND, J. 1976. A reexamination of the role of theory in psychology. Vortrag am 21. Internationalen Kongreß für Psychologie, Paris, 18.-25. Juli 1976; hektographiert.

SMEDSLUND, J. 1978a. Bandura's theory of self-efficacy: A set of common sense theorenis. Scandinavian Journal of Psychology 19, 1-14.

SmeDSLUND, J. 1978b. Some psychological theories are not empirical: Reply to Bandura. Scandinavian Journal of Psychology 19, 101-102.

SmedSLUND, J. 1980. Analyzing the primary code: From empiricism to apriorism. In: OLson, D. R. (Ed.) The social foundations of language and thought: Essays in honor of Jerome S. Bruner. New York: Norton.

Stegmüller. W. 1969. Probleme und Resultate der Wissenschaltstheoric und Analytischen Philosophie. Band II: Wissenschaftliche Frklärung und Begründung. Berlin: Springer.

Sëdmersfo, I. M. 1983. Hilfe. ich ersticke in Texten! - Eine Anleitung zur Aufarbeitung narrativer Interviews. Neue Praxis 13, 294-305.

Tausin, R. \& Tausch, A. M. 19818. Gespräachs-Psychotherapie. Einfühlsame hilfreiche Gruppen- und Einzelgespräche in Psychotherapie und alltïglichem Leben. Göttingen: Hogrefe.

TAYLOR. C. 1971. Interpretation and the sciences of man. In: RabiNov, P. \& Si:llivan, W. M. (Ed.) Interpretative social science. Berkeley: University of California Press.

Von Kutschera, F. 1972. Wissenschaftstheorie II. München: Fink.

VoN WRIGHT. G. H. 1974. Erklären und Verstehen. Franklurt a. M.: Athenäum.

Wr.kbik, H. 1971. Das Problem der Definition "aggressiver" Verhaltensweisen. Zeitschrift für Sozialpsychologie 2, 233-247.

Werbik, H. 1981. Aggression. In: WerizIK, H. \& Kaistr, H. J. (Ed.) Kritische Stichwörter zur Sozialpsychologie. München: Fink.

Wilson, T. P. 1973. Theorien der Interaktion und Modelle soziologischer Erklärung. In: Arbfitscirt ppl: Bielffelder Sozlologen (Ed.) Allagswissen. Interaktion und gesellschaftliche Wirklichkeit. Band I. Reinhek: Rowohlt. 